\title{
CONTRACTING SPACES OF MAPS ON THE COUNTABLE DIRECT LIMIT OF A SPACE
}

BY

\author{
RICHARD E. HEISEY(1)
}

ABSTRACT. We give conditions sufficient to imply the contractibility of the space of maps, with compact-open topology, on the countable direct limit of a space. Applying these conditions we obtain the following: Let $F$ be the conjugate of a separable infinite-dimensional Banach space with bounded weaktopology, or the countable direct limit of the real line. Then there is a contraction of the space of maps on $F$ which simultaneously contracts the subspaces of open maps, embeddings, closed embeddings, and homeomorphisms. Corollaries of our work are that any homeomorphism on $F, F$ as above, is invertibly isotopic to the identity, and the general linear group of the countable direct limit of lines is contractible.

0 . Introduction. Let $\left(X, x_{0}\right)$ be a pointed topological space. For each integer $n \geq 1$, let $X^{n}$ denote the cartesian product of $n$ copies of $X$, and define $j_{n}: X^{n} \rightarrow X^{n+1}$ by $j_{n}\left(x_{1}, \ldots, x_{n}\right)=\left(x_{1}, \ldots, x_{n}, x_{0}\right)$. Let $\left(X, x_{0}\right)^{\infty}$ denote the direct limit of the sequence

$$
x^{1} \stackrel{i_{1}}{\longrightarrow} x^{2} \stackrel{i_{2}}{\longrightarrow} x^{3} \stackrel{i_{3}}{\longrightarrow} \ldots
$$

Let $P\left(\left(X, x_{0}\right)^{\infty}\right)=\left\{f:\left(X, x_{0}\right)^{\infty} \rightarrow\left(X, x_{0}\right)^{\infty} \mid f\right.$ is continuous, $f\left(\left(x_{0}, x_{0}, \ldots\right)\right)=$ $\left.\left(x_{0}, x_{0}, \ldots\right)\right\}$ with the compact-open topology. In this paper we give conditions that enable us to construct a contraction on $P\left(\left(X, x_{0}\right)^{\infty}\right)$ and then investigate some of the properties possessed by this contraction. Our contraction is defined analogously to the one defined by P. L. Renz in [9] in contracting the homeomorphism group of product spaces. The direct limit case, however, requires considerable extra care in obtaining continuity of the defined contraction.

Received by the editors May 10, 1973.

AMS (MOS) subject classifications (1970). Primary 54C35, 57E05; Secondary 54D50.

Key words and phrases. Direct limit, function space, topological vector space, Banach space, bounded weak-* topology, Hilbert cube.

(1) This research was done while the author was a Rotary Foundation Fellow at the University of Warwick, England, 1971-72, and constitutes part of the author's Ph.D. thesis at Cornell University. 
Given a separable, infinite-dimensional Banach space $B$, let $B^{*}$ denote the conjugate (or dual) of $B$, and let $B^{*}\left(b^{*}\right)$ denote $B^{*}$ with its bounded weak-* topology. (The bounded weak-* topology on $B^{*}$ is the finest topology agreeing with the weak-* topology on bounded sets.) We show that $B^{*}\left(b^{*}\right)$ is homeomorphic to $Q^{\infty}$, where $Q$ is the Hilbert cube. Using this we obtain the following: Let $F$ be $B^{*}\left(b^{*}\right)$ or $R^{\infty}$, where $B$ is a separable, infinite-dimensional Banach space and $R$ is the reals. Let $C(F)=\{f: F \rightarrow F \mid f$ is continuous $\}$ with the compact-open topology. Then there is a contraction $\Psi: C(F) \times I \rightarrow C(F)$ such that $\Psi$ simultaneously contracts the following subspaces of $C(F)$ : (1) maps that take the origin to the origin, (2) open maps, (3) embeddings, (4) closed embeddings, and (5) homeomorphisms. If $F=R^{\infty}$, then $\Psi$ also contracts the subspace of linear maps. (Thus, the general linear group of $R^{\infty}$ is contractible.) Corollaries of our work are that any homeomorphism of $B^{*}\left(b^{*}\right)$ or $R^{\infty}$ is invertibly isotopic to the identity, and any linear homeomorphism of $R^{\infty}$ is invertibly isotopic to the identity through linear homeomorphisms.

The author gratefully acknowledges the advice and encouragement of his thesis advisor, David W. Henderson, and also helpful conversations with David Elworthy and James West.

I. Notation. By "space" we mean "Hausdorff topological space". By "map" we mean "continuous function". Given a space $X$, and an integer $n \geq 1$, $X^{n}$ denotes the cartesian product of $n$ copies of $X$. We let $I$ denote $[0,1], R$ the reals, and $Q$ the Hilbert cube. We will regard $Q$ as the countable product of the interval $[-1,1]$ and, when convenient, will regard $Q$ as a pointed space with base point $(0,0, \ldots)$.

Given a homotopy $\phi: X \times[a, b] \rightarrow Y$, where $X$ and $Y$ are spaces and $[a, b] \subset R$ is an interval, we denote the map $X \rightarrow Y$ given by $x \rightarrow \phi(x, t)$, for fixed $t \in[a, b]$, by both $\phi_{t}$ and $\phi(\cdot, t)$.

For any space $X$, we define the following spaces of functions, all with the compact-open topology:

$$
\begin{aligned}
& C(X)=\{f: X \rightarrow X \mid f \text { is a map }\}, \\
& O(X)=\{f \in C(X) \mid f \text { is an open map }\}
\end{aligned}
$$

(A map $f: X \rightarrow Y$ is open if it carries each open set in $X$ onto an open set in $Y$.)

$$
\begin{aligned}
E(X) & =\{f \in C(X) \mid f \text { is a embedding }\}, \\
C E(X) & =\{f \in C(X) \mid f \text { is a closed embedding }\}, \\
H(X) & =\{f \in C(X) \mid f \text { is a homeomorphism }\} .
\end{aligned}
$$

If $X=\left(X, x_{0}\right)$ is a pointed space, we let $P(X)=\left\{f \in C(X) \mid f\left(x_{0}\right)=x_{0}\right\}$, and let $O P(X), E P(X), C E P(X)$, and $H P(X)$ denote $O(X) \cap P(X), E(X) \cap P(X)$, $C E(X) \cap P(X)$ and $H(X) \cap P(X)$, respectively. 
If $F$ is a topological vector space, we regard $F$ as a pointed space with base point 0 . We let $L(F)=\{f \in C(F) \mid f$ is linear $\}$.

If $\left(X, x_{0}\right)$ is a pointed space, we define $j_{n}: X^{n} \rightarrow X^{n+1}$ (as in the introduction) by $j_{n}\left(x_{1}, \ldots, x_{n}\right)=\left(x_{1}, \ldots, x_{n}, x_{0}\right)$, and let $\left(X, x_{0}\right)^{\infty}=\operatorname{inj} \lim \left\{\left(X^{n}, j_{n}\right)\right\}$, the direct limit of the sequence

$$
x^{1} \stackrel{i_{1}}{\longrightarrow} x^{2} \stackrel{j_{2}}{\longrightarrow} x^{3} \stackrel{j_{3}}{\longrightarrow} \ldots
$$

We regard $\left(X, x_{0}\right)^{\infty}$ as consisting of all sequences $x=\left(x_{1}, x_{2}, x_{3}, \ldots\right)$ of elements of $X$ where all but finitely many of the $x_{i}$ are $x_{0}$. For each $n \geq 1$ we identify $X^{n}$ with $X^{n} \times\left\{x_{0}\right\} \times\left\{x_{0}\right\} \times \ldots \subset\left(X, x_{0}\right)^{\infty}$. Then a set $V \subset\left(X, x_{0}\right)^{\infty}$ is open if and only if $V \cap X^{n}$ is open in $X^{n}$ for every $n$. We regard $\left(X, x_{0}\right)^{\infty}$ as a pointed space with base point $\left(x_{0}, x_{0}, \ldots\right)$. Except where clarity demands otherwise, we will usually write $X^{\infty}$ for $\left(X, x_{0}\right)^{\infty}$.

If $B$ is a Banach space, $B^{*}$ will denote the conjugate (or dual) of $B$, and $B^{*}\left(b^{*}\right)$ will denote $B^{*}$ with its bounded weak-* topology.

We use the abbreviation "TVS" for "topological vector space" and "c-o" for "compact-open". We use " $\cong$ " to denote "is homeomorphic to". If $X$ is a space and $A \subset X$, then $\bar{A}$ denotes the closure of $A$ in $X$.

II. Statement of results. The following definition will be useful.

Definition. A pointed space $\left(X, x_{0}\right)$ will be said to have the direct limit product property (hereafter abbreviated DLPP) if sets of the form $\left(\Theta_{1} \times \theta_{2} \times \ldots\right)$ $\cap\left(X, x_{0}\right)^{\infty}$, where each $\mathcal{O}_{i}$ is open in $X$, form a basis for the topology of $\left(x, x_{0}\right)^{\infty}$.

Given an arbitrary pointed space $\left(X, x_{0}\right)$, the map $X^{\infty} \rightarrow X \times X^{\infty}$ given by $\left(x_{1}, x_{2}, x_{3}, \ldots\right) \mapsto\left(x_{1},\left(x_{2}, x_{3}, \ldots\right)\right)$ need not be a homeomorphism. Indeed, in the first remark in \$III we show that this is the case when $X$ is a separable Hilbert space. The proofs in this paper work only when such maps are homeomorphisms, and we insure this by hypothesizing that our spaces have the DLPP. The following proposition, proven in \$III, shows that having the DLPP is closely related to being locally compact.

Proposition II-1. (a) Any locally compact, pointed space bas the DLPP. (b) If $\left(X, x_{0}\right)$ is paracompact and first countable, then $\left(X, x_{0}\right)$ bas the DLPP if and only if $X$ is locally compact.

The basic advantage that the hypothesis of having the DLPP has for us over the hypothesis of being locally compact is that the nonlocally compact spaces $Q^{\infty}$ and $R^{\infty}$ both have the DLPP (see Corollary III-3. Note that this gives a round-about proof that $Q^{\infty}$ and $R^{\infty}$ are not first countable). Thus, in the proof of Theorem II-4 in $\S I V$ we can apply Theorem II-2 below with $X=Q^{\infty}$ and $X=R^{\infty}$. 
Definition [5, p. 248]. A space is a $k$-space if it has the weak topology determined by the family of its compact subspaces.

Note that any locally compact space is a $k$-space.

Question. What is the relationship between being a $k$-space and having the DLPP?

Definition. A homotopy $\phi: X \times[a, b] \rightarrow Y$ is an invertible isotopy if the map $\bar{\phi}: X \times[a, b] \rightarrow Y \times[a, b]$ defined by $\bar{\phi}(x, t)=(\phi(x, t), t)$ is a homeomorphism. If $\phi$ is an invertible isotopy we say $\phi_{a}$ and $\phi_{b}$ are invertibly isotopic.

Given a pointed space $\left(X, x_{0}\right)$, let $p$ denote the homeomorphism on $X^{\infty}$ given by $p\left(x_{1}, x_{2}, x_{3}, x_{4}, \ldots\right)=\left(x_{2}, x_{1}, x_{3}, x_{4}, \ldots\right)$.

Theorem II-2. Let $\left(X, x_{0}\right)$ be a pointed space having the DLPP and assume that there is a bomotopy $\phi: X^{\infty} \times I \rightarrow X^{\infty}$ with $\phi_{0}=\mathrm{id}, \phi_{1}=p$, and $\phi_{t} \in P\left(X^{\infty}\right)$ for every $t \in I$. Then

(a) there is a contraction $\Psi: P\left(X^{\infty}\right) \times 1 \rightarrow P\left(X^{\infty}\right)$ of $P\left(X^{\infty}\right)$ to the identity map;

(b) if $\phi_{t} \in O P\left(X^{\infty}\right)$, resp. $E P\left(X^{\infty}\right), C E P\left(X^{\infty}\right), H P\left(X^{\infty}\right)$ for $t \in I$, then $\Psi$ may be taken to simultaneously contract $O P\left(X^{\infty}\right)$, resp. $E P\left(X^{\infty}\right), C E P\left(X^{\infty}\right)$, $H P\left(X^{\infty}\right)$ (i.e. $\Psi\left(O P\left(X^{\infty}\right) \times I\right) \subset O P\left(X^{\infty}\right)$, etc.); and

(c) if $\phi$ is an invertible isotopy we may further obtain that $\Psi(f \circ g, t)=$ $\Psi(f, t) \circ \Psi(g, t)$ for every $t \in I$.

The proof of Theorem II-2 is given in \$III.

Now let $\left(X, x_{0}\right), \phi: X^{\infty} \times I \rightarrow X^{\infty}$ and $\Psi$ be as in Theorem II-2. Let $A$ be a space and $\alpha: A \times X^{\infty} \rightarrow X^{\infty}$ a map such that for every a $\in A, a\left(a_{0},\right) \in P\left(X^{\infty}\right)$. Define $\gamma: A \times X^{\infty} \times I \rightarrow X^{\infty}$ by $\gamma(a, x, t)=\Psi(a(a, \cdot), t)(x)$. Note that $\gamma(a, x, 0)=$ $a(a, x)$ and $\gamma(a, x, 1)=x$. Define $\bar{\alpha}: A \times X^{\infty} \rightarrow A \times X^{\infty}$ and $\bar{\gamma}: A \times X^{\infty} \times I \rightarrow$ $A \times X^{\infty} \times I$ by $\bar{\alpha}(a, x)=(a, a(a, x))$, and $\bar{\gamma}(a, x, t)=(a, \gamma(a, x, t), t)$.

Theorem II-3. Let $\left(X, x_{0}\right), \phi, A, \alpha, \gamma, \bar{\alpha}$, and $\bar{\gamma}$ be as above, and assume that either $A$ is locally compact or that $A \times X^{\infty}$ is a k-space. Then $\gamma$ is continuous. If $\phi$ is an invertible isotopy and if $\bar{\alpha}$ is an open embedding, resp. closed embedding, homeomorphism, then $\bar{\gamma}$ is an open embadding, resp. closed embedding, bomeomorphism.

Theorem II-3 is proved in $\$$ III.

Applying Theorems II-2 and II-3 to topological vector spaces, we obtain the following in \$IV.

Theorem II-4. Let $F$ be a TVS such that $F \simeq X^{\infty}$, where $X$ is a pointed, locally compact space. Then there is a contraction $\Lambda: C(F) \times I \rightarrow C(F)$ of $C(F)$ to the identity map on $F$ such that $\Lambda(f \circ g, t)=\Lambda(f, t) \circ \Lambda(g, t)$, all $g, f \in C(F)$ and $t \in I$, and such that $\Lambda$ simultaneously contracts $P(F), O(F), E(F), C E(F)$, 
and $H(F)$. Further, let $A$ be a space such that $A \times F$ is a k-space, and let $a: A \times F \rightarrow F$ be a map. Then the function 2 : $A \times F \times I \rightarrow A \times F \times I$ defined by $\mathcal{U}(a, x, t)=(a, \Lambda(a(a, \cdot), t)(x), t)$ is continuous. Define $\bar{a}: A \times F \rightarrow A \times F$ by $\bar{\alpha}(a, x)=(a, \alpha(a, x))$. If $\bar{\alpha}$ is an open embedding, resp. closed embedding, bomeomorphism, then so is $\mathcal{U}$. Finally, if $X$ is a TVS and $F=X^{\infty}$, then the contraction $\Lambda$ above also contracts $L(F)$.

Remark. The hypotheses on $F$ in Theorem II-4 imply that $F$ is a $k$-space and that $F \cong F \times F$ (by Corollary III-1 since $\left.X^{\infty} \cong(X \times X)^{\infty}\right)$. Thus, the hypothesis that $A \times F$ be a $k$-space is satisfied, for example, when $A$ is locally compact (see Lemma III-7) or when $A$ is a closed subset of $F$.

The following corollary is analogous to Lemmas 2.3 and 4.1 of [4]. Along with Theorem II- 6 below, it will be used in a future paper by the author to show that locally trivial bundles and microbundles with fiber $R^{\infty}$ or $B^{*}\left(b^{*}\right), B$ a separable, infinite-dimensional Banach space, are trivial.

Corollary II-5. Let $F$ be a TVS such that $F \cong X^{\infty}$, where $X=\left(X, x_{0}\right)$ is a pointed, locally compact space. Let $A$ be any space sucb that $A \times F$ is a $k$ space, and let $\eta: A \rightarrow I$ be continuous. If $f: A \times F \rightarrow A \times F$ is an A-preserving (i.e. $f(\{a\} \times F) \subset\{a\} \times F)$ open embedding, resp. closed embedding, bomeomorphism, then there is an A-preserving open embedding, resp. closed embedding, bomeomorphism $f_{\eta}: A \times F \rightarrow A \times F$ sucb that $\left(f_{\eta}\right)_{a}=f_{a}$ for each a $\in \eta^{-1}(0)$, and $\left(f_{\eta}\right)_{a}=$ $\mathrm{id}_{F}$ for each $a \in \eta^{-1}(1)$. If $f(a, 0)=(a, 0)$ for any $a \in A$, then $f_{\eta}(a, 0)=(a, 0)$ for that $a$.

Proof of II-5. By Theorem II-4 there is an $A \times I$ preserving open embedding, resp. closed embedding, homeomorphism, $\mathscr{U}: A \times F \times I \rightarrow A \times F \times I$ such that $\mathscr{V}(a, x, 0)=(f(a, x), 0)$ and $\mathscr{U}(a, x, 1)=(a, x, 1)$ for each $(a, x) \in A \times F$; and if $f(a, 0)=(a, 0)$ (i.e. $f(a, \cdot) \in P(F))$, then $\mathscr{P}(a, 0, t)=(a, 0, t)$. The desired open embedding, resp. closed embedding, homeomorphism, is given by $f_{\eta}(a, x)=$ $\pi \mathscr{U}(a, x, \eta(a))$, where $\pi: A \times F \times l \rightarrow A \times F$ is the projection.

It is clear that $F=R^{\infty}$ satisfies the hypotheses of Theorem II- 4 and Corollary II-5. By the following theorem this is also true for $B^{*}\left(b^{*}\right)$, where $B$ is any separable, infinite-dimensional Banach space.

Theorem II-6. If $B$ is a separable, infinite-dimensional Banach space, then $B^{*}\left(b^{*}\right)$ is bomeomorphic to $Q^{\infty}$. In particular, $B^{*}\left(b^{*}\right)$ is bomeomorpbic to a separable Hilbert space with its bounded weak topology.

Theorem II- 6 is proved in $\$ \mathrm{~V}$. We single out one corollary of Theorems II-4 and II-6. 
Corollary II-7. Let $B$ be a separable, infinite-dimensional Banach space. Then any bomeomorphism on $B^{*}\left(b^{*}\right)$ or $R^{\infty}$ is invertibly isotopic to the identity. Any linear bomeomorpbism on $R^{\infty}$ is invertibly isotopic to the identity through linear bomeomorphisms.

Proof of II-7. Let $F$ denote $B^{*}\left(b^{*}\right)$ or $R^{\infty}$. By Theorem II-6, $F \cong X^{\infty}$ where $X$ is a pointed, locally compact space. It follows easily that $F$ is a $k$-space. By Theorem II -4 any homeomorphism $\alpha: F \times\{0\} \rightarrow F \times\{0\}$ extends to an I-preserving homeomorphism $\mathfrak{U}: F \times l \rightarrow F \times l$ such that $\mathfrak{U}_{1}=\mathrm{id}_{F}$. If $F=R^{\infty}$, Theorem II $\mathcal{A}$ shows that $\mathfrak{U}_{t}$ will be linear if $a$ is linear.

III. Proofs of Proposition II-1 and Theorems II-2 and II-3.

Proof of Proposition II-1. (a) Assume $\left(X, x_{0}\right)$ is locally compact. Let $a=$ $\left(a_{1}, \ldots, a_{n}, x_{0}, x_{0}, \ldots\right) \in X^{\infty}$, and let $\mathcal{O}$ be any open neighborhood of $a$ in $X^{\infty}$. Since $X^{n}$ is locally compact, there exist $\Theta_{1}, \ldots, \Theta_{n}$ open in $X$ such that

$$
\left(a_{1}, \cdots, a_{n}\right) \in \Theta_{1} \times \cdots \times \theta_{n} \subset \bar{\theta}_{1} \times \cdots \times \bar{\theta}_{n} \subset \Theta \cap X^{n}
$$

and $\overline{\mathcal{O}}_{1} \times \cdots \times \overline{\mathcal{O}}_{n}$ is compact. Fix $k \geq n$, and suppose we have

$$
\left(a_{1}, \cdots, a_{k}\right) \in \Theta_{1} \times \cdots \times \Theta_{k} \subset \bar{\theta}_{1} \times \cdots \times \bar{\theta}_{k} \subset \Theta_{\cap} x^{k} \text {, }
$$

each $\mathcal{O}_{i}$ a relatively compact open set in $x$. For every $x \in \bar{\Theta}_{1} \times \cdots \times \overline{\mathcal{O}}_{k}$ there are neighborhoods $V_{x}$ and $U_{x}$ of $x$ and $x_{0}=a_{k+1}$, resp., such that $U_{x}$ is relatively compact and $V_{x}^{x} \times \bar{U}_{x} \subset \mathcal{x} \mathcal{O} \cap X^{k+1}$. Finitely many of the $V_{x}$ 's, say $v_{x_{1}}, \ldots, v_{x_{r}}$, cover $\overline{\mathcal{O}}_{1} \times \cdots \times \overline{\mathcal{O}}_{k}$. Let $\mathcal{O}_{k+1}=\bigcap\left\{U_{x_{i}} \mid i=1, \ldots, r\right\}$. Then $\mathcal{O}_{k+1}^{x_{1}}$ is a relatively compact open neighborhood of $x_{0}$ in $X$, and $\left(a_{1}, \ldots, a_{k+1}\right)$ $\epsilon \mathcal{O}_{1} \times \cdots \times \mathcal{O}_{k+1} \subset \overline{\mathcal{O}}_{1} \times \cdots \times \overline{\mathcal{O}}_{k+1} \subset \mathcal{O} \cap X^{k+1}$. By induction we obtain $\left\{\mathcal{O}_{i} \mid i=1,2, \ldots\right\}$ such that $a \in\left(\mathcal{\Theta}_{1} \times \mathcal{O}_{2} \times \ldots\right) \cap x^{\infty} \subset \mathcal{O}$.

(b) We need only prove the "only if" assertion. So assume $X$ is not locally compact. Then there exsits an $a \in X$ such that $a$ has no relatively compact neighborhoods. Since $X$ is regular and first countable, there is a basis of open sets $\left\{V_{i} \mid i=1,2, \ldots\right\}$ at $a$ and $a$ basis of open sets $\left\{W_{i} \mid i=1,2, \ldots\right\}$ at $x_{0}$ such that $\bar{V}_{i+1} \subset V_{i}$ and $\bar{W}_{i+1} \subset W_{i}, i=1,2, \ldots$ Fix $i$. Since $\bar{V}_{i}$ is paracompact and not compact, it is not countably compact [5, p. 230]. Thus, by the Bolzano-Weierstrass property, there is a sequence $\left\{x_{j}^{i} \mid j=1,2, \ldots\right\} \subset \bar{V}_{i}$ with no cluster (or accumulation) point. Choose $w_{i} \in W_{i} \backslash\left\{x_{0}\right\}$. Let

$$
A_{i}=\left\{\left.\left(x_{j}^{i}, x_{0}, x_{0}, \ldots, x_{0}, w_{j}, x_{0}, x_{0}, \ldots\right)\right|_{j}=1,2, \ldots\right\} \subset X^{\infty},
$$

where the $w_{j}$ is in the $(i+1)_{\text {st }}$ coordinate. Let $A=\bigcup\left\{A_{i} \mid i=1,2, \ldots\right\}$.

We will show that $A$ is closed in $X^{\infty}$. It is enough to show that $A \cap X^{n}$ is closed in $X^{n}$ for each $n$. Clearly $A \cap X^{1}=\varnothing$ is closed in $X^{1}$. So let $n>1$, and let $\left(x_{1}, \ldots, x_{n}\right) \in X^{n} \backslash A$. Suppose first that $x_{i} \neq x_{0}$, some $i$ with 
$2 \leq i \leq n$. Let $U$ and $\mathcal{O}$ be neighborhoods of $x_{i}$ and $x_{0}$, respectively, such that $U \cap \overline{\mathcal{O}}=\varnothing$. Then $U^{\prime}=X \times \cdots \times X \times U \times X \times \cdots \times X, U$ the $i$ th factor, is a neighborhood of $\left(x_{1}, \ldots, x_{n}\right)$ in $X^{n}$, and $U^{\prime} \cap A \subset A_{i-1}$. Since $w_{k} \in \mathcal{O}$ for all sufficiently large $k$, we thus have $U^{\prime} \cap A$ is finite. Hence, $U^{\prime} \backslash\left(U^{\prime} \cap A\right)$ is a neighborhood of $\left(x_{1}, \ldots, x_{n}\right)$ in $X^{n}$ disjoint from $A \cap X^{n}$. Now suppose $x_{i}=x_{0}, i=2, \ldots, n$. Then, since each $\left\{x_{j}^{i} \mid j=1,2, \ldots\right\}$ has no cluster point, there is a neighborhood $G$ of $x_{1}$ such that $G \cap\left(\bigcup\left\{x_{j}^{i} \mid i=1, \ldots, n-1, j=\right.\right.$ $1,2, \ldots\})$ is either empty or $\left\{x_{s}^{r}=x_{1}\right\}$, some $r \leq n-1, s<\infty$. Find $j$ such that $w_{s} \notin W_{j}$. Then $G \times X \times \cdots \times X \times W_{j} \times X \times \cdots \times X, W_{j}$ the $(r+1)$ st factor, is a neighborhood of $x$ missing $A \cap X^{n}$. It follows that $A \cap X^{n}$ is closed, each $n \geq 1$.

We now show that this implies $X$ cannot have the DLPP. If $X$ had the DLPP, then sets of the form $\left(U_{1} \times U_{2} \times \ldots\right) \cap X^{\infty}$, where each $U_{i}$ is open in $X$ and $x_{0} \in U_{i}, i \geq 2$, form a basis at $\left(a, x_{0}, x_{0}, \ldots\right)$. But, given any such neighborhood of $\left(a, x_{0}, x_{0}, \ldots\right)$, we can find $r$ such that $a \in V_{r} \subset V_{1}$ and then an $s$ such that $w_{s} \in U_{r+1}$. Then

$$
\left(x_{s}^{r}, x_{0}, \ldots, x_{0}, w_{s}, x_{0}, \ldots\right) \in A \cap\left[\left(U_{1} \times U_{2} \times \cdots\right) \cap X^{\infty}\right] .
$$

Thus, if $X$ had the DLPP, we would have $\left(a, x_{0}, x_{0}, \ldots\right) \in \bar{A} \backslash A$, contradicting the fact that $A$ is closed.

Remark. In the proof of (b) above, suppose $X$ were a separable Hilbert space, $l_{2}$. If $\left\{e_{1}, e_{2}, \ldots\right\}$ is the standard orthonormal basis of $l_{2}$, we could take, in our proof, $a=0, V_{i}=w_{i}=\left\{x \in l_{2} \mid\|x\|<1 / i\right\}, x_{j}^{i}=(1 / 2 i) e_{j}$, and $w_{i}=(1 / 2 i) e_{i}$. Our proof then shows that the resulting set $A$ is closed in $l_{2}^{\infty}$. Let $\beta: l_{2}^{\infty} \rightarrow$ $l_{2} \times l_{2}^{\infty}$ be the natural (continuous) bijection $\left(x_{1}, x_{2}, x_{3}, \ldots\right) \rightarrow\left(x_{1},\left(x_{2}, x_{3}, \ldots\right)\right)$. It is easy to check that $(0,(0,0, \ldots))$ is in the closure of $\beta(A)$, so that $\beta(A)$ is not closed in $l_{2} \times l_{2}^{\infty}$. Thus, $\beta$ is not a homeomorphism. As indicated in $\S$ II, the arguments of this paper require that such maps $X^{\infty} \rightarrow X \times X^{\infty}$ be homeomorphisms, and we insure this by hypothesizing that $X$ has the DLPP.

The following corollary of II-1 has also been proved by R. Palais in [8, P. 120].

Corollary III-1. If $\left(X, x_{0}\right)$ and $\left(Y, y_{0}\right)$ are pointed, locally compact spaces, then $\alpha:\left(X \times Y,\left(x_{0}, y_{0}\right)\right)^{\infty} \rightarrow\left(X, x_{0}\right)^{\infty} \times\left(Y, y_{0}\right)^{\infty}$ given by

$$
\alpha\left(\left(x_{1}, y_{1}\right),\left(x_{2}, y_{2}\right), \ldots\right)=\left(\left(x_{1}, x_{2}, \ldots\right),\left(y_{1}, y_{2}, \ldots\right)\right)
$$

is a bomeomorphism.

Proof of III-1. Clearly $\alpha$ is a bijection. Since $X, Y$, and $X \times Y$ are locally compact, these spaces also have the DLPP by Proposition II-1. Using this it is easy to check that $\alpha$ is a homeomorphism. 
Corollary III-2. If $\left(X, x_{0}\right)$ is locally compact, then $O \subset\left(X^{\infty}\right)^{n}$ is open iff $\mathcal{O} \cap\left(X^{k}\right)^{n}$ is open in $\left(X^{k}\right)^{n}$, all k.

Proof of III-2. By III-1 and induction the map $\alpha:\left(X^{n}\right)^{\infty} \rightarrow\left(X^{\infty}\right)^{n}$ given by $\alpha\left(\left(\left(x_{1_{1}}, \ldots, x_{n_{1}}\right),\left(x_{1_{2}}, \ldots, x_{n_{2}}\right), \ldots\right)\right)=\left(\left(x_{1_{1}}, x_{1_{2}}, \ldots\right), \ldots,\left(x_{n_{1}}, x_{n_{2}}, \ldots\right)\right)$

is a homeomorphism. Thus $\mathcal{O} \subset\left(X^{\infty}\right)^{n}$ is open in $\left(X^{\infty}\right)^{n} \Leftrightarrow a^{-1}(\theta)$ is open in $\left(X^{n}\right)^{\infty} \Leftrightarrow a^{-1}(\mathcal{O}) \cap\left(X^{n}\right)^{k}$ is open in $\left(X^{n}\right)^{k}$ for each $k \Leftrightarrow \mathcal{O} \cap a\left[\left(X^{n}\right)^{k}\right]$ is open in $a\left[\left(X^{n}\right)^{k}\right]$ for each $k \Leftrightarrow \mathcal{O} \cap\left(X^{k}\right)^{n}$ is open in $\left(X^{k}\right)^{n}$, each $k$.

Corollary III-3. If $\left(X, x_{0}\right)$ is locally compact, then $X^{\infty}$ bas the DLPP, and $\left(X^{\infty}\right)^{\infty}$ is bomeomorpbic to $X^{\infty}$.

Proof of III-3. Using Cantor's diagonalization we obtain a bijection $\beta: X^{\infty}$ $\rightarrow\left(X^{\infty}\right)^{\infty}$,

$$
\beta\left(\left(x_{1}, x_{2}, \ldots\right)\right)=\left(\left(x_{1}, x_{3}, x_{6}, x_{10}, \ldots\right),\left(x_{2}, x_{5}, x_{9}, \ldots\right),\left(x_{4}, x_{8}, \ldots\right), \ldots\right)
$$

Then $\beta$ is continuous, since $\beta$ restricted to $X^{n}$ is continuous, each $n$. For each $n$, let $\beta_{n}^{-1}$ denote $\beta^{-1}$ restricted to $\left(X^{\infty}\right)^{n}$. Then $\beta^{-1}$ will be continuous if each $\beta_{n}^{-1}$ is continuous. By Corollary III-2, $\beta_{n}^{-1}$ will be continuous if $\beta_{n}^{-1}$ restricted to $\left(X^{k}\right)^{n}$ is continuous for each $k$. But this is clear, since Corollary III-2 also implies that $\left(X^{k}\right)^{n}$, as a subspace of $\left(X^{\infty}\right)^{\infty}$, has the natural product topology determined by $X$. Thus, $\left(X^{\infty}\right)^{\infty} \cong X^{\infty}$.

To see that $X^{\infty}$ has the DLPP, note first that $X$ has the DLPP by Proposition II-1. Thus, sets of the form $\left(U_{1} \times U_{2} \times \cdots\right) \cap X^{\infty}, U_{i}$ open in $X$, form a basis for $X^{\infty}$. But $\beta$ carries each such set to a set of the form $\left(W_{1} \times W_{2} \times \ldots\right)$ $\cap\left(X^{\infty}\right)^{\infty}$, where each $W_{i}$ is open in $X^{\infty}$. Since $\beta$ is a homeomorphism, these sets must form a basis for $\left(X^{\infty}\right)^{\infty}$ as required.

Remark. Thus, for example, $Q^{\infty}$ and $R^{\infty}$ have the DLPP, $\left(Q^{\infty}\right)^{\infty} \simeq Q^{\infty}$ and $\left(R^{\infty}\right)^{\infty} \simeq R^{\infty}$.

Lemma III-4. If $\psi: S \times[0,1] \rightarrow S$ is a bomotopy of a space $S$, then $(f, t)$ $\rightarrow \psi(., t) \circ f$ and $(f, t) \rightarrow f \circ \psi(., t)$ are bomotopies $C(S) \times I \rightarrow C(S)$.

Proof of III-4. The proof of the lemma given by Renz in [9] applies to this more general situation, provided we change " $g(K) \subset \bigcap\left\{V_{k} \mid k \in K_{0}\right\}$ " to read " $g(K) \subset \bigcup\left\{V_{k} \mid k \in K_{0}\right\} "$.

Lemma III-5. Given spaces $\left(X, x_{0}\right), Y, Z$ such that $Y$ is locally compact, $f: X^{\infty} \times Y \rightarrow Z$ is continuous if and only if $f$ restricted to $X^{k} \times Y$ is continuous for every $k$. 
Proof of III-5. The "only if" assertion is immediate. Suppose $f$ is continuous on $X^{k} \times Y$ for every $k$. Define $f^{\prime}: X^{\infty} \rightarrow Z^{Y}$ by $f^{\prime}(x)(y)=f(x, y)$. Here $Z^{Y}$ denotes the function space of maps $Y \rightarrow Z$ with the c-o topology. By [5, 3.1(1), p. 261], $f^{\prime}: X^{k} \rightarrow Z^{Y}$ is continuous. Thus, $f^{\prime}$ is continuous. Since $Y$ is locally compact, by $\left[5,3.1(2)\right.$, p. 261] we have $f: X^{\infty} \times Y \rightarrow Z$ is continuous.

Lemma III-6. Given $\left(X, x_{0}\right)$, any compact subset of $X^{\infty}$ is contained in $X^{n}$, some $n$.

Proof of III-6. Let $K \subset X^{\infty}$ be such that $K \not \subset X^{n}$, any $n$. Then there is a sequence $\left\{n_{i}\right\}$ with $n_{i+1}>n_{i}$ and a sequence $\left\{k_{i}\right\}$ such that $k_{i} \in X^{n_{i}} \backslash X^{n_{i-1}}$. Then $\left\{k_{i}\right\}$ has no limit point. By the Bolzano-Weierstrass property $K$ cannot be compact.

Proof of Theorem II-2. We will define the contraction on $P\left(X^{\infty}\right) \times[0, \infty]$ rather than on $P\left(X^{\infty}\right) \times I$.

For $n \geq 1$ defined $\pi^{n}: X^{\infty} \rightarrow X^{\infty}$ by

$$
\pi^{n}(x)=\left(x_{1}, \ldots, x_{n-1}, x_{n+1}, x_{n+2}, \cdots\right) \text {, }
$$

where $x=\left(x_{1}, x_{2}, \cdots\right)$, and $\rho_{n}: X^{\infty} \rightarrow X \times X^{\infty}$ by

$$
\rho_{n}(x)=\left(x_{n}, \pi^{n}(x)\right)
$$

Since $X$ has the DLPP, it is clear that $\rho_{n}$ is a homeomorphism. Given $f \in P\left(X^{\infty}\right)$ and $n \geq 1$, define $f^{n}$ by $f^{n}=\rho_{n}^{-1}(\mathrm{id} \times f) \rho_{n} \in P\left(X^{\infty}\right)$. Explicitly,

$$
f^{n}(x)=\left(f\left(\pi^{n}(x)\right)_{1}, \ldots, f\left(\pi^{n}(x)\right)_{n-1}, x_{n}, f\left(\pi^{n}(x)\right)_{n}, \ldots\right),
$$

from which it is easily seen that our $f^{n}$ is analogous to the one used by Renz in [9]. We show that $f \rightarrow f^{n}$ is a continuous map of $P\left(X^{\infty}\right)$ into $P\left(X^{\infty}\right)$. Let $K \subset X^{\infty}$ be compact, and let $U=\left(U_{1} \times U_{2} \times \cdots\right) \cap X^{\infty}$ be a basic open subset of $X^{\infty}$. Let $P(K, U)=\left\{f \in P\left(X^{\infty}\right) \mid f(K) \subset U\right\}$. Then sets of the form $P(K, U)$ form a subbasis for the c-o topology of $P\left(X^{\infty}\right)$ (see [5, p. 264]). Let $f^{n} \in P(K, U)$. Let $\pi_{1}: X \times X^{\infty} \rightarrow X$ and $\pi_{2}: X \times X^{\infty} \rightarrow X^{\infty}$ be projections. Note that

$$
f^{n} \in P(K, U) \Rightarrow(\mathrm{id} \times f) \rho_{n}(K) \subset \rho_{n}(U) \Rightarrow \pi_{1} \rho_{n}(K) \subset \pi_{1} \rho_{n}(U) .
$$

Thus, if $g \in P\left(\pi_{2} \rho_{n}(K), \pi_{2} \rho_{n}(U)\right)$, then (id $\left.\times g\right)\left(\rho_{n}(K)\right) \subset \rho_{n}(U)$, so that $g^{n} \in P(K, U)$. It follows that $f \rightarrow f^{n}$ is continuous.

Let $\phi: X^{\infty} \times l \rightarrow X^{\infty}$ be the hypothesized homotopy with $\phi_{0}=$ id, $\phi_{1}=p$. For every $n \geq 1$, let $T_{n}: X^{\infty} \rightarrow X^{\infty}$ be given by $T_{n}(x)=\left(x_{n}, x_{n+1}, \ldots\right)$, and let $\phi^{n}: X^{\infty} \times[n, n+1]^{n} \rightarrow X^{\infty}$ be given by $\phi^{n}(x, t)^{n}=\left(x_{1}, \ldots, x_{n-1}\right.$, $\left.\phi\left(T_{n}(x), t-n\right)\right)$. Since $X$ has the DLPP, $T_{n}$ and $\phi^{n}$ are continuous. Define $\Phi: P\left(X^{\infty}\right) \times[1, \infty] \rightarrow P\left(X^{\infty}\right)$ by 


$$
\Phi(f, t)=\left\{\begin{array}{l}
\phi^{n}(\cdot, t) \circ f^{n} \circ \phi^{n}(\cdot, t), \quad f \in P\left(X^{\infty}\right), t \in[n, n+1] \\
f, \quad t=\infty .
\end{array}\right.
$$

Note that

$$
\phi^{n}(\cdot, n+1) \circ f^{n} \circ \phi^{n}(\cdot, n+1)=f^{n+1}=\phi^{n+1}(\cdot, n+1) \circ f^{n+1} \circ \phi^{n+1}(\cdot, n+1)
$$

and

$$
\Phi(f, t) \in P\left(X^{\infty}\right),
$$

so that $\Phi$ is well-defined. Since $f \rightarrow f^{n}$ is continuous, $\Phi$ restricted to $P\left(X^{\infty}\right) \times$ $[1, \infty)$ will be continuous provided $\left(f^{n}, t\right) \rightarrow \Phi(f, t)$ is continuous. But $\left(f^{n}, t\right) \rightarrow$ $\Phi(f, t)$ is the composite of $(f, t) \mapsto\left(\phi^{n}(., t) \circ f, t\right)$ and $(g, t) \rightarrow g \circ \phi^{n}(\cdot, t)$, both of which are continuous by Lemma III-4.

We proceed to show that $\Phi$ is continuous at $\infty$. Let $f=\Phi(f, \infty) \in P(K, U)$, where $K \subset X^{\infty}$ is compact and $U=\left(U_{1} \times U_{2} \times \ldots\right) \cap X^{\infty}$ is a basic open set in $X^{\infty}$. We find (and this is sufficient) a neighborhood of $(f, \infty)$ in $P\left(X^{\infty}\right) \times[1, \infty]$ that is mapped by $\Phi$ into $P(K, U)$. Note, by Lemma III-6, that $K \cup f(K) \subset X^{N}$ for some $N$. Then $f(K) \subset X^{N} \cap U$ implies that $x_{0} \in U_{n}$ for $n \geq N+1$. Let

$$
U^{\prime}=\left(U_{1} \times \cdots \times U_{N} \times U_{N+1} \cap U_{N+2} \times U_{N+2} \cap U_{N+3} \times \cdots\right) \cap X^{\infty} .
$$

Note that $f \in P\left(K, U^{\prime}\right)$.

We continue via a series of steps:

(i) For nonnegative $t$, let $[t]$ be the greatest integer less than or equal to $t$. Define $r: X^{\infty} \times[1, \infty] \rightarrow X^{\infty}$ by

$$
\tau(x, t)= \begin{cases}\pi^{[t]} \circ \phi^{[t]}(x, t), & t<\infty, \\ x, & t=\infty .\end{cases}
$$

Fix $k \geq 1$. Since $\pi^{n} \phi^{n}(x, n+1)=\pi^{n+1}(x)$, we see that $r=\pi^{n} \circ \phi^{n}$ on $X^{\infty} \times$ $[n, n+1]$, so that $\tau$ is continuous on $X^{\infty} \times[1, \infty)$. If $t>k+1$, then $T$ is the identity on $X^{k} \times\{t\}$, so $r$ is continuous on $X^{k} \times(k+1, \infty]$. Hence $r$ is continuous on $X^{k} \times[1, \infty]$, each $k$. By Lemma III-5, $r$ is continuous. Thus, by Lemma III-4 the map $A: P\left(X^{\infty}\right) \times[1, \infty] \rightarrow P\left(X^{\infty}\right)$ given by $A(g, t)=r(\cdot, t) \circ g$ is continuous. Since $A(f, \infty)=f \in P\left(K, U^{\prime}\right)$, there is an open set $\Theta \subset P\left(X^{\infty}\right)$ and an integer $M_{1}$, such that $f \in \mathcal{O}$ and $A\left(\mathcal{O}_{\times}\left[M_{1}, \infty\right]\right) \subset P\left(K, U^{\prime}\right)$.

(ii) Let $K^{1}, \ldots, K^{r}$ be compact subsets and $U^{1}, \ldots, U^{r}$ basic open subsets of $X^{\infty}$ such that $f \in \bigcap\left\{P\left(K^{i}, U^{i}\right) \mid i=1, \ldots, r\right\} \subset \mathcal{O}$. By Lemma III-6 we may choose an integer $M_{2}$ such that $K^{i} \cup f\left(K^{i}\right) \subset X^{M_{2}}, i=1, \ldots, r$. Writing $U^{i}=$ $\left(U_{1}^{i} \times U_{2}^{i} \times \cdots\right) \cap X^{\infty}$ let 


$$
\left(U^{i}\right)^{\prime}=\left(U_{1}^{i} \times \cdots \times U_{M_{2}}^{i} \times U_{M_{2}+1}^{i} \cap U_{M_{2}+2}^{i} \times U_{M_{2}+2}^{i} \cap U_{M_{2}+3}^{i} \times \cdots\right) \cap X^{\infty} .
$$

Note that $f \dot{\epsilon} P\left(K^{i},\left(U^{i}\right)^{\prime}\right), i=1, \ldots, r$. Let $n \geq M_{2}+1$ and $g \in P\left(K^{i},\left(U^{i}\right)^{\prime}\right)$. Then, for $k \in K^{i}$,

$$
\begin{aligned}
g^{n}(k) & =\left(g \pi^{n}(k)_{1}, \cdots, g \pi^{n}(k)_{n-1}, k_{n}, g \pi^{n}(k)_{n}, \ldots\right) \\
& =\left(g(k)_{1}, \cdots, g(k)_{n-1}, x_{0}, g(k)_{n}, \ldots,\right) \in U^{i},
\end{aligned}
$$

so that $g^{n}\left(K^{i}\right) \subset U^{i}$. Thus, letting $V=\bigcap\left\{P\left(K^{i},\left(U^{i}\right)^{\prime}\right) \mid i=1, \ldots, r\right\}$, we have $V$ open in $P\left(X^{\infty}\right), f \in V$, and if $g \in V$ and $n \geq M_{2}+1$, then $g^{n} \in \mathcal{O}$.

(iii) Let $M=\max \left\{M_{1}, M_{2}+1, N+1\right\}$. Let $n \geq M$ and $(g, t) \in V \times[n, n+1]$. Then $g^{n} \in \mathcal{O}$, so $A\left(g^{n}, t\right) \in P\left(K, U^{\prime}\right)$. Thus, for every $k \in K$,

$$
\begin{aligned}
\tau\left(g^{n}(k), t\right) & =\pi^{n} \phi^{n}\left(\left(g \pi^{n}(k)_{1}, \cdots, g \pi^{n}(k)_{n-1}, k_{n}, g \pi^{n}(k)_{n}, \cdots\right), t\right) \\
& =\pi^{n}\left(g(k)_{1}, \cdots, g(k)_{n-1}, \phi\left(\left(x_{0}, T_{n}(g(k))\right), t-n\right)\right) \\
& =\left(g(k)_{1}, \cdots, g(k)_{n-1}, \pi^{1} \phi\left(\left(x_{0}, T_{n}(g(k))\right), t-n\right)\right) \in U^{\prime} .
\end{aligned}
$$

(iv) Define $\psi: X^{\infty} \times[0,1] \rightarrow X^{\infty}$ by

$$
\psi(x, t)=\left(\phi\left(\left(x_{0}, T_{1}(x)\right), t\right)_{1}, \phi\left(\left(x_{0}, T_{2}(x)\right), t\right)_{1}, \phi\left(\left(x_{0}, T_{3}(x)\right), t\right)_{1}, \ldots\right) .
$$

Note that since $T_{n}(x)$ is eventually $\left(x_{0}, x_{0}, \ldots\right)$ for any given $x$, and since $\phi\left(x_{0}, t\right)=x_{0}$, we have $\psi(x, t) \in X^{\infty}$. Using Lemma III-5, $\psi$ is easily seen to be continuous. Define $\mathcal{U}: P\left(X^{\infty}\right) \times I \rightarrow P\left(X^{\infty}\right)$ by $\mathcal{U}(g, t)=\psi(\cdot, t) \circ g$. By Lemma III-4 $\mathscr{U}$ is continuous. Since $f(K) \subset X^{N}$, we see that

$$
\mathcal{U}(f \times l) \subset P\left(K,\left(X^{N} \times U_{N+1} \times U_{N+2} \times \cdots\right) \cap X^{\infty}\right) \text {. }
$$

Thus, there is an open set $W$ in $P\left(X^{\infty}\right)$ such that $f \in W$ and

$$
\mathscr{U}(W \times I) \subset P\left(K,\left(X^{N} \times U_{N+1} \times U_{N+2} \times \cdots\right) \cap X^{\infty}\right) .
$$

(v) We have $f \in V \cap W \cap P(K, U)$, which is open in $P\left(X^{\infty}\right)$. We show that $\Phi(V \cap W \cap P(K, U) \times[M, \infty]) \subset P(K, U)$. Let $t \geq M, g \in V \cap W \cap P(K, U)$. If $t=\infty$, then $\Phi(g, t)=g \in P(K, U)$. So assume $t \in[n, n+1]$, where $n \geq M$. Then $\Phi(g, t)=\phi^{n}(\cdot, t) \circ g^{n} \circ \phi^{n}(., t)$. Let $k \in K \subset X^{N}$. Then $\phi^{n}$ restricted to $X^{N}$ is the identity, so

$$
\begin{aligned}
\Phi(g, t)(k) & =\phi^{n}(\cdot, t)\left(g^{n}(k)\right)=\phi^{n}(\cdot, t)\left(g(k)_{1}, \cdots, g(k)_{n-1}, x_{0}, g(k)_{n}, \ldots\right) \\
& =\left(g(k)_{1}, \cdots, g(k)_{n-1}, \phi\left(\left(x_{0}, T_{n}(g(k))\right), t-n\right)\right) .
\end{aligned}
$$

Since $g \in V$ and $n \geq M$, we know from step (iii) that

$$
\left(g(k)_{1}, \cdots, g(k)_{n-1}, \pi^{1} \phi\left(\left(x_{0}, T_{n}(g(k))\right), t-n\right)\right) \in U^{\prime} \text {. }
$$


By definition of $U^{\prime}$ we will thus have $\Phi(g, t)(k) \in U$ provided $\phi\left(\left(x_{0}, T_{n}(g(k))\right), t-n\right)_{1}$ $\epsilon U_{n^{*}}$. But this is true from step (iv), since $g \in W$ and $n \geq N+1$.

This completes the proof of the continuity of $\Phi: P\left(X^{\infty}\right) \times[1, \infty] \rightarrow P\left(X^{\infty}\right)$. Note that $\Phi(f, 1)=f^{1}$ and $\Phi(f, \infty)=f$. For $n \geq 1$, let $P^{n}\left(X^{\infty}\right)=\left\{f \in P\left(X^{\infty}\right) \mid f(x)_{i}=\right.$ $\left.x_{i}, i \leq n, x \in X^{\infty}\right\}$. Let $P^{0}\left(X^{\infty}\right)=P\left(X^{\infty}\right)$. For $n \geq 1$, it is routine to check that $\Phi\left(P^{n}\left(X^{\infty}\right) \times[n+1, \infty]\right) \subset P^{n}\left(X^{\infty}\right)$. Since, for $n \geq 0, \Phi(f, n+1)=f^{n+1}$, by reparametrizing $\Phi$ restricted to $P^{n}\left(X^{\infty}\right) \times[n+1, \infty]$ we obtain a homotopy

$$
\Phi^{n}: P^{n}\left(X^{\infty}\right) \times[n, n+1] \rightarrow P^{n}\left(X^{\infty}\right), \quad n \geq 0,
$$

such that $\Phi^{n}(f, n)=f, \Phi^{n}(f, n+1)=f^{n+1}$.

For $n \geq 1$, let $\lambda_{n}: X^{\infty} \rightarrow X^{n} \times X^{\infty}$ be the homeomorphsim $\lambda_{n}(x)=\left(\left(x_{1}, \ldots\right.\right.$, $\left.\left.x_{n}\right), T_{n+1}(x)\right)$, and define ${ }^{n} f=\lambda_{n}^{-1} \circ(\mathrm{id} \times f) \circ \lambda_{n}$. More explicitly, $\left({ }^{n} f\right)(x)=$ $\left(x_{1}, \ldots, x_{n}, f\left(x_{n+1}, x_{n+2}, \ldots\right)\right)$. With $\%=f$, it is easy to prove by induction that ${ }^{n+1} f=\left({ }^{n} f\right)^{n+1}, n \geq 0$. Define $\Psi: P\left(X^{\infty}\right) \times[0, \infty] \rightarrow P\left(X^{\infty}\right)$ by

$$
\Psi(f, t)= \begin{cases}\Phi^{n}(n f, t), & t \in[n, n+1], \\ \text { identity, } & t=\infty .\end{cases}
$$

Since ${ }^{n} f \in P^{n}\left(X^{\infty}\right)$ and $\Phi^{n}(n f, n+1)=\left({ }^{n} f\right)^{n+1}={ }^{n+1} f$, we see that $\Psi$ is well defined. To show that $\Psi$ restricted to $P\left(X^{\infty}\right) \times[0, \infty)$ is continuous, it is sufficient to prove that $f \rightarrow{ }^{n} f$ is continuous as a map of $P\left(X^{\infty}\right)$ into $P\left(X^{\infty}\right)$. But, since $f \rightarrow f^{n}$ is continuous, this is an easy inductive argument.

We proceed to show that $\Psi$ is continuous at $\infty$. Let $P(K, U)$ be a subbasic neighborhood of id in $P\left(X^{\infty}\right)$, where $K$ is compact and $U=\left(U_{1} \times U_{2} \times \ldots\right) \cap X^{\infty}$. Since id $\epsilon P(K, U), K \subset U$. By Lemma III-6, $K \subset U \cap X^{N}$, some N. Fix $n \geq N$, and let $f \in P\left(X^{\infty}\right), t \in(n, n+1]$, and $k \in K$. Then,

$$
\begin{aligned}
\Psi(f, t)(k) & =\Phi^{n}\left({ }^{n} f, t\right)(k)=\Phi\left({ }^{n} f, t^{\prime}\right)(k), \text { where } t^{\prime} \in[j, j+1], j \geq n+1 \geq N+1, \\
& =\phi^{j}\left(\circ, t^{\prime}\right) \circ\left({ }^{n} f\right)^{j} \circ \phi^{j}\left(\cdot, t^{\prime}\right)(k) \\
& =\phi^{j}\left(\cdot, t^{\prime}\right)\left({ }^{n} f(k)_{1}, \ldots,{ }^{n} f(k)_{n-I},{ }^{n} f(k)_{n}, \ldots\right), \text { since } k \in X^{N}, \\
& =\phi^{j}\left(\cdot, t^{\prime}\right)\left(k_{1}, \ldots, k_{N}, x_{0}, x_{0}, \cdots\right)=k .
\end{aligned}
$$

Thus, $\Psi\left(P\left(X^{\infty}\right) \times(N, \infty]\right) \subset P(K, U)$, and $\Psi$ is continuous at $\infty$. Since $\Psi(f, 0)=$ $\Phi^{0}(0,0)=f, \Psi$ contracts $P\left(X^{\infty}\right)$ to id, and we have proven (a) of Theorem II-2.

Statement (b) of Theorem II-2 follows from the observation that if $\phi(\cdot, t)$ and $f$ are elements of $O P\left(X^{\infty}\right)$, resp. $E P\left(X^{\infty}\right), C E P\left(X^{\infty}\right), H P\left(X^{\infty}\right)$, then so are $\phi^{n}(\cdot, t), f^{n}=\rho_{n}^{-1} \circ(\mathrm{id} \times f) \circ \rho_{n}$ and ${ }^{n} f=\lambda_{n}^{-1} \circ(\mathrm{id} \times f) \circ \lambda_{n} \cdot$

To prove statement (c) of Theorem II-2, as sume $\phi: X^{\infty} \times I \rightarrow X^{\infty}$ is an invertible isotopy. Then the map $(x, t) \mapsto \phi(., t)^{-1}(x)$ is continuous. In this case, modify the definition of $\Phi$ in the proof above to 


$$
\Phi(f, t)= \begin{cases}\phi^{n}(\cdot, t)^{-1} \circ f^{n} \circ \phi^{n}(\cdot, t), & t \in[n, n+1], \\ f, & t=\infty .\end{cases}
$$

Note that $\phi^{n}(\cdot, t)^{-1}(x)=\left(x_{1}, \ldots, x_{n-1}, \phi(\cdot, t)^{-1}\left(T_{n}(x)\right)\right)$, which is continuous in $x$ and $t$. One proceeds to check that $\Phi$ is continuous as in the original proof with the following changes: In step (i) change the definition of $r$ to

$$
\tau(x, t)= \begin{cases}\pi^{[t]} \phi^{[t]}(\cdot, t)^{-1}(x), & t<\infty, \\ x, & t=\infty .\end{cases}
$$

At the end of step (iii) one obtains

$$
\left(g(k)_{1}, \cdots, g(k)_{n-1}, \pi^{1}\left[\phi(\cdot, t-n)^{-1}\left(\left(x_{0}, T_{n} g(k)\right)\right)\right]\right) \in U^{\prime} .
$$

Then in step (iv) define

$$
\psi(x, t)=\left(\left[\phi(\cdot, t)^{-1}\left(\left(x_{0}, T_{1}(x)\right)\right)\right]_{1},\left[\phi(\cdot, t)^{-1}\left(\left(x_{0}, T_{2}(x)\right)\right)\right]_{1}, \ldots\right) .
$$

Since $(f \circ g)^{n}=f^{n} \circ g^{n}$, this new $\Phi$ satisfies $\Phi(f \circ g, t)=\Phi(f \circ t) \circ \Phi(g, t)$. Now construct $\Psi$ from the "new" $\Phi$ as before. Since ${ }^{n}(f \circ g)={ }^{n} f \circ{ }^{n} g, \Psi$ will be a contraction such that $\Psi(f \circ g, t)=\Psi(f, t) \circ \Psi(g, t)$. Clearly, this $\Psi$ will also simultaneously contract the spaces $O P\left(X^{\infty}\right), E P\left(X^{\infty}\right), C E P\left(X^{\infty}\right)$ and $H P\left(X^{\infty}\right)$.

This completes the proof of Theorem II-2.

In preparation for the proof of Theorem II-3 we establish a few additional lemmas.

Lemma III-7 [5, p. 263]. Let $X$ be a k-space and $Y$ a locally compact space. Then $X \times Y$ is a k-space.

Lemma III-8. Let $\left(X, x_{0}\right)$ be a pointed space, and let $Y$ be a space sucb that either $Y$ is locally compact or $Y \times X^{\infty}$ is a k-space. Then a set $C \subset Y \times X^{\infty}$ is open, resp. closed, in $Y \times X^{\infty}$ if and only if it is open, resp. closed, in $Y \times X^{k}$ for every $k \geq 1$.

Proof of III-8. Assume first that $Y$ is locally compact. Let $r_{1}$ denote the product topology on $Y \times X^{\infty}$, and let $r_{2}$ denote the topology on $Y \times X^{\infty}$ obtained by regarding $Y \times X^{\infty}$ as the direct limit of the $Y \times X^{k}$ 's. We are to show id: $\left(Y \times X^{\infty}, r_{1}\right) \rightarrow\left(Y \times X^{\infty}, r_{2}\right)$ is a homeomorphism. Clearly id is open. By Lemma III-5 id will be continuous if it is continuous restricted to each $Y \times X^{k}$. This is clear:

Assume now that $Y \times X^{\infty}$ is a $k$-space. By Lemma II-6, any compact subspace of $Y \times X^{\infty}$ is contained in $Y \times X^{k}$, some $k$, and the assertion of Lemma III-8 follows. 
Lemma III-9. Let $f \in P\left(X^{\infty}\right)$, and let $f^{n}$ be defined as in the proof of Theorem II-2. Then, if $n \geq k+1,\left(\operatorname{Im} f^{n}\right) \cap X^{k}=(\operatorname{Im} f) \cap X^{k}$.

Proof of III-9. Let $\rho_{n}: X^{\infty} \rightarrow X \times X^{\infty}$ be as in the proof of Theorem II-2. Then, for $n \geq k+1$, we have

$$
\begin{aligned}
\left(\operatorname{Im} f^{n}\right) \cap X^{k} & =\left[\operatorname{Im}\left(\rho_{n}^{-1} \circ(\mathrm{id} \times f) \circ \rho_{n}\right)\right] \cap X^{k}=\left[\rho_{n}^{-1}\left(X \times f\left(X^{\infty}\right)\right)\right] \cap X^{k} \\
& =\left[\rho_{n}^{-1}\left(\left\{x_{0}\right\} \times f\left(X^{\infty}\right)\right)\right] \cap X^{k}=f\left(X^{\infty}\right) \cap X^{k} .
\end{aligned}
$$

Proof of Theorem II-3. Identify $I$ with $[0, \infty]$, and let $\Psi: P\left(X^{\infty}\right) \times[0, \infty] \rightarrow$ $P\left(X^{\infty}\right)$ be the contraction constructed in the proof of Theorem II-2. Recall

$$
\Psi(f, t)= \begin{cases}\Phi^{n}\left({ }^{n} f, t\right), & t \in[n, n+1], \\ \mathrm{id}, & t=\infty .\end{cases}
$$

We are to prove $\gamma: A \times X^{\infty} \times[0, \infty] \rightarrow X^{\infty}$ defined by $\gamma(a, x, t)=\Psi(a(a, \cdot), t)(x)$ is continuous. By Lemmas III-7 and III-8 it is sufficient to show that $\gamma$ restricted to $A \times X^{k} \times[0, \infty]$ is continuous for every $k$. Fix $k$. Let $n \geq 0$ and $t \in[n, n+1]$. Then $\gamma(a, x, t)=\Phi^{n}(n(a(a, \cdot)), t)(x)$. Recall that $\Phi^{n}$ is a reparametrization of $\Phi$ restricted to $P\left(X^{\infty}\right) \times[n+1, \infty]$. Thus, there is a homeomorphism $\delta=\delta_{n}$ : $[n, n+1] \rightarrow[n+1, \infty]$ with $\delta(n)=\infty$ and $\delta(n+1)=n+1$ such that $\Phi^{n}(n(\alpha(a, \cdot)), t)=\Phi\left({ }^{n}(\alpha(a, \cdot)), \delta(t)\right)$. Then, for $t \in \delta^{-1}([j, j+1]), j \geq n+1$, we have

$$
\gamma(a, x, t)=\left(\phi^{j}(\cdot, \delta(t)) \circ\left(^{n} \alpha(a, \cdot)\right)^{j}\right)\left(\phi^{j}(x, \delta(t))\right) .
$$

This will be continuous in $a, x$, and $t$ provided the map $(a, x) \mapsto\left({ }^{n} \alpha(a, \cdot)\right)^{j}(x)$ is a continuous map of $A \times X^{\infty}$ into $X^{\infty}$. But

$$
\left({ }^{n} \alpha(a, \cdot)\right)^{j}(x)=\rho_{j}^{-1}\left(\mathrm{id} \times \lambda_{n}^{-1}(\mathrm{id} \times \alpha(a, \cdot)) \lambda_{n}\right) \rho_{j}(x)
$$

which is jointly continuous in $a$ and $x$. Thus, for each $n \geq 0, \gamma$ is continuous on $A \times X^{k} \times(n, n+1]$.

We proceed to show that $\gamma$ is continuous at $n$. Let $(b, y, n) \in A \times X^{k} \times\{n\}$, and let $\gamma(b, y, n) \in U=\left(U_{1} \times U_{2} \times \cdots\right) \cap X^{\infty}$. Choose $N \geq k$ such that $\gamma(b, y, n)$ $\epsilon X^{N}$. Then, for each $i>N, U_{i}$ is a neighborhood of $x_{0}$. Hence $\gamma(b, y, n) \in U^{\prime}$, where

$$
U^{\prime}=\left(U_{1} \times \cdots \times U_{N} \times U_{N+1} \cap U_{N+2} \times U_{N+2} \cap U_{N+3} \times \cdots\right) \cap X^{\infty} .
$$

Define $r: X^{\infty} \times[1, \infty] \rightarrow X^{\infty}$ as in step (i) in the proof of Theorem II-2. It was shown there that $r$ is continuous. Define $\bar{\delta}: X^{\infty} \times[n, n+1] \rightarrow X^{\infty} \times[n+1, \infty]$ by $\bar{\delta}(x, t)=(x, \delta(t))$. Since $\tau \bar{\delta}\left({ }^{n} \alpha(b,).(y), n\right)={ }^{n} \alpha(b,).(y)=\gamma(b, y, n) \in U^{\prime}$, there is a neighborhood $V=\left(V_{1} \times V_{2} \times \ldots\right) \cap X^{\infty}$ of ${ }^{n} a(b, \cdot)(y)$ in $X^{\infty}$ and an $\epsilon_{1}>0$ such that $\tau \bar{\delta}\left(V \times\left[n, n+\epsilon_{1}\right)\right) \subset U^{\prime}$. Note that 


$$
{ }^{n} a(b, \cdot)(y) \in V^{\prime}=\left(V_{1} \times \cdots \times V_{N} \times V_{N+1} \cap V_{N+2} \times V_{N+2} \cap V_{N+3} \times \cdots\right) \cap X^{\infty} \text {. }
$$

Define $\eta: A \times X^{k} \rightarrow X^{\infty}$ by

$$
\eta(a, x)={ }^{n} a(a, \cdot)(x)=\left(x_{1}, \ldots, x_{n}, a\left(a,\left(x_{n+1}, x_{n+2}, \ldots\right)\right)\right) .
$$

Then $\eta$ is continuous, and $\eta(b, y) \in V^{\prime}$. Thus, there are neighborhoods $\Theta_{1}$ of $b$ in $A$ and $\mathcal{O}_{2}$ of $y$ in $X^{k}$ such that $\eta\left(\mathcal{O}_{1} \times \mathcal{O}_{2}\right) \subset V^{\prime}$. As before, let [t] denote the greatest integer not greater than $t$. Choose $\epsilon<\epsilon_{1}$ so small that if $t \epsilon$ $[n, n+\epsilon)$, then $[\delta(t)] \geq N+1$. Now let $(a, x, t) \epsilon \mathcal{O}_{1} \times \mathcal{O}_{2} \times(n, n+\epsilon) \subset A \times X^{k} \times$ $(n, n+\epsilon)$. To simplify notation, let $r=[\delta(t)]$. Now $(a, x) \in \mathcal{O}_{1} \times \mathcal{O}_{2}$ implies ${ }^{n} a(a,).(x) \in V^{\prime}$. Since $r \geq N+1 \geq k+1$, this implies $\left({ }^{n} a(a, \cdot)\right)^{r}(x) \in V$. This implies

$$
r \circ \bar{\delta}\left(\left({ }^{n} \alpha(a, \cdot)\right)^{r}(x), t\right)=\pi^{r} \phi^{r}\left(\left({ }^{n} \alpha(a, \cdot)\right)^{r}(x), \delta(t)\right) \in U^{\prime} .
$$

Since $\gamma(a, x, t)=\phi^{r}\left(\left({ }^{n} a(a, \cdot)\right)^{r}(x), \delta(t)\right)$, we will thus have $\gamma(a, x, t) \in U$ provided

$$
\left.\left[\phi^{r}\left(\left({ }^{n} \alpha(a, \cdot)\right)\right)^{r}(x), \delta(t)\right)\right]_{r}=\phi\left(\left(x_{0}, T_{r}\left({ }^{n} \alpha(a, \cdot)(x)\right)\right), \delta(t)-r\right)_{1} \in U_{r}
$$

Define $\xi: A \times X^{k} \times I \rightarrow X^{\infty}$ by

$$
\xi(a, x, t)=\left(\phi\left(\left(x_{0}, T_{1}\left({ }^{n} \alpha(a, .)(x)\right)\right), t\right)_{1}, \phi\left(\left(x_{0}, T_{2}\left({ }^{(n} \alpha(a, \cdot)(x)\right)\right), t\right), \ldots\right) .
$$

Then $\xi=\psi(\eta(a, x), t)$, where $\psi$ is as defined in step (iv) in the proof of Theorem II-2, so $\xi$ is continuous. Since $\xi(b, y, t) \in\left(X^{N} \times U_{N+1} \times U_{N+2} \times \cdots\right) \cap X^{\infty}$ for every $t \in I$, there are neighborhoods $\Theta_{1}^{\prime} \subset \mathcal{O}_{1}$ of $b$ in $A$ and $\Theta_{2}^{\prime} \subset \Theta_{2}$ of $y$ in $X^{k}$ such that $\xi\left(\Theta_{1}^{\prime} \subset \mathcal{O}_{2}^{\prime} \times I\right) \subset\left(X^{N} \times U_{N+1} \times U_{N+2} \times \cdots\right) \cap X^{\infty}$. It follows that $\gamma\left(\Theta_{1}^{\prime} \times \Theta_{2}^{\prime} \times[n, n+\epsilon)\right) \subset U$. Hence $\gamma$ is continuous at $n$ and therefore on all of $A \times X^{k} \times[0, \infty)$.

To see that $\gamma$ is continuous at $t=\infty$, simply note that $\gamma$ restricts to the identity on $A \times X^{k} \times(k, \infty)$. This completes the proof that $\gamma$ is continuous.

Note (see the proof of Theorem II-2(c)) that if $\phi$ is an invertible isotopy, and if $\psi$ is constructed using $\Phi(f, t)=\phi^{n}(\cdot, t)^{-1} \circ f^{n} \circ \phi^{n}(\cdot, t)$, a proof similar to the one above establishes that $\gamma$ is continuous. The basic change is to redefine $r$ as was done in the proof of statement (c) in Theorem II-2.

Now assume further that $\phi$ is an invertible isotopy and that $\bar{\alpha}$ is an open embedding. We are to show that $\bar{\gamma}$ is an open embedding. Again we identify $I$ with $[0, \infty]$. Let $\Psi: P\left(X^{\infty}\right) \times[0, \infty] \rightarrow P\left(X^{\infty}\right)$ be the contraction constructed in the proof of (c) in Theorem II-2. That is, we assume $\Psi$ is constructed from $\Phi$, where $\Phi(f, t)=\phi^{n}(\cdot, t)^{-1} \circ f^{n} \circ \phi^{n}(\cdot, t)$. (The proof is easily altered if the "original" $\Psi$ constructed in the proof of Theorem II-2 is used.) We already know $\gamma$, and hence $\bar{\gamma}$, is continuous.

We show first that $\operatorname{Im} \bar{\gamma}$ (the image of $\bar{\gamma}$ ) is open in $A \times X^{\infty} \times[0, \infty]$. Fix 
$k \geq 1$. By Lemmas III-7 and III-8 it is sufficient to show that $(\operatorname{Im} \bar{\gamma}) \cap\left(A \times X^{k} \times\right.$ $[0, \infty])$ is open in $A \times X^{k} \times[0, \infty]$. This will be true if we can show

(a) $(\operatorname{Im} \bar{\gamma}) \cap\left(A \times X^{k} \times(n, n+1]\right)$ is open in $A \times X^{k} \times(n, n \times 1], n \geq 0$;

(b) if $n \geq 0$ and $(b, z, n) \in(\operatorname{Im} \bar{\gamma}) \cap\left(A \times X^{k} \times\{n\}\right)$, then $\operatorname{Im} \bar{\gamma}$ contains a neighborhood of $(b, z, n)$ in $A \times X^{k} \times[n, n+1]$; and

(c) $\operatorname{Im} \bar{\gamma} \supset A \times X^{k} \times(k, \infty]$.

To prove (a), let $\delta_{n}:[n, n+1] \rightarrow[n+1, \infty]$ be the homeomorphism used to parametrize $\Phi$ restricted to $[n+1, \infty]$ to obtain $\Phi^{n}$. Recall that $\delta_{n}(n)=\infty$ and $\delta_{n}(n+1)=n+1$. To simplify notation, let $n(t)=\delta_{n}(t)$ for $t \in[n, n+1]$. Then, for $t \in \delta_{n}^{-1}([j, j+1])$,

$$
\bar{\gamma}(a, x, t)=\left(a, \phi^{j}(\cdot, n(t))^{-1}\left({ }^{n} \alpha(a, \cdot)\right)^{j} \phi^{j}(\cdot, n(t))(x), t\right) .
$$

Note that $\phi$ an invertible isotopy and $\bar{\alpha}$ open imply that the following maps are open:

$$
\begin{aligned}
(a, x, t) & \rightarrow\left(a, \phi^{j}(\cdot, n(t))(x), t\right), \\
(a, x, t) & \rightarrow\left(a,\left(^{n} \alpha(a, \cdot)\right)^{j}(x), t\right) \\
& =\left(a, \rho_{j}^{-1}\left(\mathrm{id} \times \lambda_{n}^{-1}(\mathrm{id} \times a(a, \cdot)) \lambda_{n}\right) \rho_{j}(x), t\right),
\end{aligned}
$$

and

$$
(a, x, t) \rightarrow\left(a, \phi^{j}(\cdot, n(t))^{-1}(x), t\right)
$$

Thus, $\bar{\gamma}$ restricted to $A \times X^{\infty} \times \delta_{n}^{-1}([j, j+1])$ is the composite of open maps, and (a) follows.

To prove (b), let $(b, z, n)=\bar{\gamma}(b, y, n) \in A \times X^{k} \times\{n\}$. Then $z=\left({ }^{n} \alpha(b,).\right)(y)$. Since the map $A \times X^{\infty} \rightarrow A \times X^{\infty}$ given by

$$
(a, x) \rightarrow\left(a,\left({ }^{n} \alpha(a, \cdot)\right)(x)\right)=\left(a, \lambda_{n}^{-1}(\mathrm{id} \times a(a, \cdot)) \lambda_{n}(x)\right)
$$

is open, there are neighborhoods $W_{1}$ of $b$ in $A$ and $W_{2}$ of $z$ in $X^{k}$ such that for every $a \in W_{1},\left({ }^{n} a(a, \cdot)\right)\left(X^{\infty}\right) \supset W_{2}$. Let $\epsilon>0$ be so small that $\delta_{n}([n, n+\epsilon]) \subset$ $(k+1, \infty]$. Then, for every $(a, t) \in W_{1} \times(n, n+\epsilon)$,

$$
\begin{aligned}
\gamma\left(\{a\} \times X^{\infty} \times\{t\}\right) & =\phi^{[n(t)]}(\cdot, n(t))^{-1}\left({ }^{n} a(a, \cdot)\right)^{[n(t)]} \phi^{[n(t)]}(\cdot, n(t))\left(X^{\infty}\right) \\
& \left.\supset\left[\left(^{n} a(a, \cdot)\right)\right)^{[n(t)]}\left(X^{\infty}\right)\right] \cap X^{k} .
\end{aligned}
$$

By Lemma III-9 this last set equals $\left[\left(^{n} a(a,).\right)\left(X^{\infty}\right)\right] \cap X^{k}$. Thus, $(b, z, n) \epsilon$ $W_{1} \times W_{2} \times[n, n+\epsilon) \subset \operatorname{Im} \bar{\gamma}$.

Finally, for (c), simply note that $\bar{\gamma}$ restricted to $A \times X^{k} \times(k, \infty]$ is the identity. Thus, $\operatorname{Im} \bar{\gamma}$ is open. 
We now show that $\bar{\gamma}^{-1}: \operatorname{Im} \bar{\gamma} \rightarrow A \times X^{\infty} \times[0, \infty]$ is continuous. Since $\operatorname{Im} \bar{\gamma}$ is open, it follows easily from Lemmas III-7 and III- 8 that $\bar{\gamma}^{-1}$ will be continuous provided it is continuous when restricted to $(\operatorname{Im} \bar{\gamma}) \cap\left(A \times X^{k} \times[0, \infty]\right)$, each $k \geq 1$. For each $n \geq 0$, let $\delta_{n}:[n, n+1] \rightarrow[n+1, \infty]$ and $n(t)=\delta_{n}(t)$ be as before. Again let $[t]$ denote the greatest integer not greater than $t$. For $t \in$ $(n, n+1]$ we have

$$
\begin{aligned}
\gamma(a, x, t) & =\Psi(\alpha(a, \cdot), t)(x) \\
& =\phi^{[n(t)]}(\cdot, n(t))^{-1} \circ\left(^{n} \alpha(a, \cdot)\right)^{[n(t)]} \circ \phi^{[n(t)]}(\cdot, n(t))(x) .
\end{aligned}
$$

Now, if $f \in E P\left(X^{\infty}\right)$, then $f^{n}=\rho_{n}^{-1}(\mathrm{id} \times f) \rho_{n}$ and ${ }^{n} f=\lambda_{n}^{-1}(\mathrm{id} \times f) \lambda_{n}$ are also in $E P\left(X^{\infty}\right)$, and, on theit respective domains, $\left(f^{n}\right)^{-1}=\left(f^{-1}\right)^{n}$ and $\left(n^{n} f\right)^{-1}={ }^{n}\left(f^{-1}\right)$. Thus, for every $(a, x, t) \in \bar{\gamma}\left(A \times X^{\infty} \times[0, \infty]\right), \bar{\gamma}^{-1}(a, x, t)=(a, \zeta(a, x, t), t)$ where, for $t \in(n, n+1]$,

$$
\zeta(a, x, t)=\phi^{[n(t)]}(\cdot, n(t))^{-1} \circ\left(n\left(a(a, \cdot)^{-1}\right)\right)^{[n(t)]} \circ \phi^{[n(t)]}(\cdot, n(t))(x),
$$

and $\zeta(a, x, n)=\left(n\left(\alpha(a, \cdot)^{-1}\right)\right)(x)$.

Proceeding as with $\gamma, \zeta$ is shown continuous on $(\operatorname{dom} \zeta) \cap\left(A \times X^{k} \times(n, n+1]\right)$, for every $n \geq 0$. To prove that $\zeta$ is continuous at $n$, again proceed as before using the definition of $r: X^{\infty} \times[n, n+1] \rightarrow X^{\infty}$ appropriate for our $\Psi$ and defining $\eta$ : dom $\eta \subset A \times X^{k} \rightarrow X^{\infty}$ by $\eta(a, x)={ }^{n}\left(a(a, \cdot)^{-1}\right)(x)$, where for every $a \in A$,

$$
(\operatorname{dom} \eta) \cap\left(\{a\} \times X^{k}\right)=\{a\} \times \operatorname{dom}\left({ }^{n}\left(a(a, \cdot)^{-1}\right)\right) \cap X^{k}=\{a\} \times\left(\operatorname{Im}{ }^{n} a(a, \cdot)\right) \cap X^{k} .
$$

Again $\eta$ is continuous, and there are neighborhoods $\Theta_{1}$ of $b$ in $A$ and $\mathcal{O}_{2}$ of $y$ in $X^{k}$ such that $\eta\left((\operatorname{dom} \eta) \cap\left(\mathcal{O}_{1} \times \mathcal{O}_{2}\right)\right) \subset V^{\prime}$. Choose $\epsilon<\epsilon_{1}$ as before, and let $(a, x, t) \epsilon\left(\Theta_{1} \times \Theta_{2} \times[n, n+\epsilon)\right) \cap \operatorname{dom} \zeta=\left(\Theta_{1} \times \mathcal{\Theta}_{2} \times[n, n+\epsilon)\right) \cap \operatorname{Im} \bar{\gamma}$. To proceed, we now need to know that

$$
(a, x, t) \in\left(\mathcal{O}_{1} \times \mathcal{O}_{2} \times[n, n+\epsilon)\right) \cap \operatorname{dom} \zeta \Rightarrow(a, x) \epsilon(\operatorname{dom} \eta) \cap\left(\Theta_{1} \times \mathcal{O}_{2}\right) .
$$

To see this, observe that $(a, x, t) \in \operatorname{Im} \bar{\gamma}$ implies, provided $t \neq n$ (the case $t=n$ is easier), that

$$
x=\phi^{[n(t)]}(\cdot, n(t))^{-1} \circ(n \alpha(a, \cdot))^{[n(t)]} \circ \phi^{[n(t)]}(\cdot, n(t))(z),
$$

some $z \in X^{\infty}$. Since $n(t) \geq N+1 \geq k+1$, and $x \in X^{k}$,

$$
\left.x=\left({ }^{n} a(a, \cdot)\right)\right)^{[n(t)]} \circ \phi^{[n(t)]}(\cdot, n(t))(z) \in \operatorname{Im}\left(\left({ }^{n} a(a, \cdot)\right)\right)^{[n(t)]} \cap X^{k} \text {. }
$$

By Lemma III-9, we thus have $x \in \operatorname{Im}\left({ }^{n} a(a, \cdot)\right) \cap X^{k}$. Thus, $(a, x) \in(\operatorname{dom} \eta) \cap$ $\left(\mathcal{O}_{1} \times \mathcal{O}_{2}\right)$ as required. Again, proceed as before, defining $\xi:\left(A \times X^{k} \times I\right) \cap$ $\operatorname{dom} \xi \rightarrow X^{\infty}$ by 
$\left.\xi(a, x, t)=\left(\phi(\cdot, t)^{-1}\left(x_{0}, T_{1}{ }^{(n}\left(\alpha(a, \cdot)^{-1}\right)(x)\right)\right)_{1}, \phi(\cdot, t)^{-1}\left(x_{0}, T_{2}{ }^{(n}\left(a(a, \circ)^{-1}\left(x_{0}\right)\right)\right)_{1}, \ldots\right)$, where $(\operatorname{dom} \xi) \cap\left(\{a\} \times X^{k} \times\{t\}\right)$ is $\{a\} \times \operatorname{dom}\left({ }^{n}\left(\alpha(a, .)^{-1}\right)\right) \cap X^{k} \times\{t\}$ : As before we obtain neighborhoods $\mathcal{O}_{1}^{\prime} \subset \mathcal{O}_{1}$ of $b$ and $\theta_{2}^{\prime} \subset \mathcal{O}_{2}$ of $y$ such that $\xi\left((\operatorname{dom} \xi) \cap\left(\mathcal{O}_{1}^{\prime} \times \mathcal{O}_{2}^{\prime} \times I\right)\right) \subset\left(X^{N} \times U_{N+1} \times U_{N+2} \times \cdots\right) \cap X^{\infty}$. As done for $\eta$ above, one checks that $(a, x, t) \in(\operatorname{Im} \bar{\gamma}) \cap\left(\mathcal{O}_{1}^{\prime} \times \mathcal{O}_{2}^{\prime} \times[n, n+\epsilon)\right)$ implies $(a, x, t) \in \operatorname{dom} \xi$. The proof of the continuity of $\zeta$, and hence $\bar{\gamma}^{-1}$, is then completed following the proof given for $\gamma$.

Now assume that $\phi$ is an invertible isotopy and that $\bar{\alpha}$ is a closed embedding. We show first that $\operatorname{Im} \bar{\gamma}$ is closed in $A \times X^{\infty} \times[0, \infty]$. Fix $k \geq 1$. Again, by Lemmas III-7 and III-8, it is sufficient to show that $(\operatorname{Im} \bar{\gamma}) \cap\left(A \times X^{k} \times[0, \infty]\right)$ is closed in $A \times X^{k} \times[0, \infty]$. To show this it is sufficient to show

(d) $(\operatorname{Im} \bar{\gamma}) \cap\left(A \times X^{k} \times(n, n+1]\right)$ is closed in $A \times X^{k} \times(n, n+1], n \geq 0$,

(e) for each $n>0$, if $\left\{\left(a_{i}, y_{i}, t_{i}\right)=\bar{\gamma}\left(a_{i}, x_{i}, t_{i}\right) \mid i \in 9\right\}$ is a net in Im $\bar{\gamma} n$ $\left(A \times X^{k} \times[n, n+1]\right)$ indexed by $g$ and converging to $(a, y, n) \in A \times X^{k} \times\{n\}$, then $(a, y, n) \in \operatorname{Im} \bar{\gamma}$, and

(f) $\operatorname{Im} \bar{\gamma} \supset A \times X^{k} \times(k, \infty)$.

The proof of (d) is analogous to the proof of (a) above, and we omit it. Condition ( $f$ ) is the same as (c), which has already been established.

To prove (e), fix $n \geq 0$, and choose $\epsilon>0$ small enough so that $\delta_{n}([n, n+\epsilon])$ $C(k+1, \infty]$. Let $\left\{\left(a_{i}, \bar{y}_{i}, t_{i}\right)=\bar{\gamma}\left(a_{i}, x_{i}, t_{i}\right) \mid i \in 9\right\}$ be a net in $(\operatorname{Im} \bar{\gamma}) \cap$ $\left(A \times X^{k} \times[n, n+\epsilon]\right)$ indexed by $\mathscr{I}$ and converging to $(a, y, n) \in A \times X^{k} \times\{n\}$. It suffices to show that $(a, y, n) \in \operatorname{Im} \bar{\gamma}$. For $i \in \mathcal{G}$, let $j_{i}=\left[n\left(t_{i}\right)\right]$. Note that $j_{i} \geq k+1$. Letting $\zeta_{i}=\phi^{j_{i}}\left(x_{i}, n\left(t_{i}\right)\right)$, and using that $y_{i} \in X^{k}$, we have

$$
y_{i}=\phi^{j}\left(\cdot, n\left(t_{i}\right)\right)^{-1}\left({ }^{n} \alpha\left(a_{i}, \cdot\right)\right)^{j}{ }_{i}\left(\zeta_{i}\right)=\left({ }^{n} \alpha\left(a_{i}, \cdot\right)\right)^{j}{ }_{i}\left(\zeta_{i}\right)=\left({ }^{n} \alpha\left(a_{i}, \cdot\right)\right)\left(\pi^{j}{ }^{i}\left(\zeta_{i}\right)\right) .
$$

Thus, $\left(a_{i},\left(n^{n} a\left(a_{i},.\right)\right)\left(\pi^{j}\left(\zeta_{i}\right)\right)\right) \rightarrow(a, y)$. But $\bar{\alpha}$ a closed embedding implies that the map $(b, x) \mapsto\left(b,\left({ }^{n} a(b,).\right)(x)\right)$ is closed. Thus, $(a, y)=\left(a,\left({ }^{n} a(a,).\right)(\zeta)\right)$, some $\zeta \in X^{\infty}$, and $(a, y, n)=\bar{\gamma}(a, \zeta, n) \in \operatorname{Im} \bar{\gamma}$ as required.

Thus, $\operatorname{Im} \bar{\gamma}$ is closed in $A \times X^{\infty} \times[0, \infty]$. The proof that $\bar{\gamma}^{-1}: \operatorname{Im} \bar{\gamma} \rightarrow A \times$ $X^{\infty} \times[0, \infty]$ is continuous is the same as the proof given for the case when $\bar{\alpha}$ was an open embedding.

Assume, finally, that $\bar{\alpha}$ is a homeomorphism. Then $\bar{\gamma}$ is onto since $\Psi$ contracts $H P\left(X^{\infty}\right)$ (see Theorem II-2). This completes the proof of Theorem II-3.

A remark on the countable product case. If $X$ is any space we can formulate analogues of Theorems II-2 and II-3 for the space $X^{\omega}$, the countable product of the space $X$. Here, of course, the hypothesis that $X$ has the DLPP is not needed. If $\phi: X^{\omega} \times I \rightarrow X^{\omega}$ is a homotopy between the identity and the map $\left(x_{1}, x_{2}, x_{3}, x_{4}, \ldots\right) \rightarrow\left(x_{2}, x_{1}, x_{3}, x_{4}, \ldots\right)$, then one can construct a 
contraction $\Psi: C\left(X^{\omega}\right) \times I \rightarrow C\left(X^{\omega}\right)$ such that if each $\phi_{t}$ is in $O\left(X^{\omega}\right)$, resp. $E\left(X^{\omega}\right), C E\left(X^{\omega}\right), H\left(X^{\omega}\right)$, then $\Psi$ simultaneously contracts $O\left(X^{\omega}\right)$, resp. $E\left(X^{\omega}\right)$, $C E\left(X^{\omega}\right), H\left(X^{\omega}\right)$. If also $X=\left(X, x_{0}\right)$ is pointed, and if $\phi_{t} \in P\left(X^{\omega}\right)$ for each $t$, then $\Psi$ also contracts $P\left(X^{\omega}\right)$. (Here $X^{\omega}$ is regarded as a pointed space with base point $\left(x_{0}, x_{0}, \ldots\right)$.) The proof of the above assertion amounts to observing that the contraction constructed by Renz in [9], when extended to $C\left(X^{\omega}\right)$, satisfies these conditions.

Turning to the analogue of Theorem II-3, let $X, \phi, \Psi$ be as in the above paragraph. Let $A$ be any space, and $\alpha: A \times X^{\omega} \rightarrow X^{\omega}$ any map. Define

$$
\gamma: A \times X^{\omega} \times I \rightarrow X^{\omega}, \quad \bar{\alpha}: A \times X^{\omega} \rightarrow A \times X^{\omega}, \quad \bar{\gamma}: A \times X^{\omega} \times I \rightarrow A \times X^{\omega} \times I
$$

by

$$
\gamma(a, x, t)=\Psi(a(a, \cdot), t)(x), \quad \bar{a}(a, x)=(a, a(a, x)), \quad \gamma(a, x, t)=(a, \gamma(a, x, t), t) .
$$

Then $\gamma$ is continuous. The proof of this is again essentially in [9]. If $\phi$ is an invertible isotopy, and if $\bar{\alpha}$ is a closed embedding, resp. homeomorphism, then arguments similar to those in the proof of Theorem II-3 show that $\bar{\gamma}$ is a closed embedding, resp. homeomorphism.

If $\bar{a}$ is an open embedding, however, $\bar{\gamma}$ need not be an open embedding (in the product case). We illustrate by an example. Let $X=R$. It is known (for instance, the proof of Lemma IV-1 is easily adopted) that there is an invertible isotopy $\phi: R^{\omega} \times I \quad R^{\omega}$ with $\phi_{0}=$ id and $\phi_{1}\left(x_{1}, x_{2}, x_{3}, x_{4} \cdots\right)=$ $\left(x_{2}, x_{1}, x_{3}, x_{4}, \ldots\right)$. In the analogue of Theorem II-3 above, let $A$ be a point, identify $A \times R^{\omega}$ with $R^{\omega}$, and let $\alpha: R^{\omega} \rightarrow R^{\omega}$ be an open embedding that takes the first factor of $R$ onto $(-1,1)$, so that $\alpha\left(R^{\omega}\right)=(-1,1) \times R \times R \times \cdots$. Let $\Psi: C\left(R^{\omega}\right) \times[0, \infty] \rightarrow C\left(R^{\omega}\right)$ be the contraction analogous to the one constructed in the proof of Theorem II-3, and let $\bar{\gamma}: R^{\omega} \times I \rightarrow R^{\omega} \times I$ be defined by $\bar{\gamma}(x, t)=$ $(\Psi(a, t)(x), t)$. Then $\bar{\gamma}$ is not open: If $\bar{\gamma}$ were open, then, since $(0, \infty)=$ $\bar{\gamma}(0, \infty) \in \operatorname{Im} \bar{\gamma}$, we would have a neighborhood $U=U_{1} \times \cdots \times U_{N} \times \Pi_{i>N} R$ and an integer $k$ such that $(U \times(k, \infty]) \subset \operatorname{Im} \bar{\gamma}$. But then for every $n>k$ (notation as in the proof of Theorem II-3), $\bar{\gamma}\left(R^{\omega} \times\{n\}\right)=\left({ }^{n} a\right)\left(R^{\omega}\right) \times\{n\} \supset U \times\{n\}$. This is impossible since $\left({ }^{n} \alpha\right)\left(R^{\omega}\right)=\Pi_{1}^{n} R \times(-1,1) \times \Pi_{i \geq n+2} R$.

IV. The topological vector space case (Theorem II-4). If $F$ is a TVS, we regard $F^{\infty}=\operatorname{inj} \lim F^{n}$ as a TVS with coordinate-wise addition and scalar multiplication.

Lemma IV-1. Let $F$ be a TVS. Then there is an invertible isotopy $\psi: F^{\infty} \times$ $I \rightarrow F^{\infty}$ such that $\psi_{0}=\mathrm{id}, \psi_{1}\left(x_{1}, x_{2}, x_{3}, x_{4}, \ldots\right)=\left(x_{2}, x_{1}, x_{3}, x_{4}, \ldots\right)$, and $\psi_{t} \in L\left(F^{\infty}\right), t \in I$. 
Proof of IV-l. For $n \geq 1$ define $g^{n}: F^{\infty} \times[0,1] \rightarrow F^{\infty}$ by

$$
g^{n}(x, t)=\left(x_{1}, \ldots, x_{n-1},(1-t) x_{n}+t x_{n+1},(1-t) x_{n+1}-t x_{n}, x_{n+2}, x_{n+3}, \ldots\right)
$$

where $x=\left(x_{1}, x_{2}, x_{3}, \ldots\right)$.

It follows easily from Lemma III-5 that $g^{n}$ is an invertible isotopy. Let $b^{1}=g^{1}$, and for $n \geq 2$ let $b^{n}: F^{\infty} \times I \rightarrow F^{\infty}$ be the invertible isotopy $b^{n}(x, t)=$ $\left(g_{t}^{n} \circ g_{t}^{n}\right)(x)$. Note that $b_{0}^{1}(x)=x_{1} b_{1}^{1}(x)=\left(x_{2},-x_{1}, x_{3}, x_{4}, \ldots\right)$, and, for $n \geq 2$, $b_{0}^{n}(x)=x$ and $b_{1}^{n}(x)=\left(x_{1}, \ldots, x_{n-1},-x_{n},-x_{n+1}, x_{n+2}, \ldots\right)$. Let $b^{0}=\mathrm{id}$, and define $\psi: F^{\infty} \times[0, \infty] \rightarrow F^{\infty}$ by

$$
\psi(x, t)= \begin{cases}b^{n+1}\left(b_{1}^{n} \circ \ldots \circ b_{1}^{0}(x), t-n\right), & t \in[n, n+1], \\ \left(x_{2}, x_{1}, x_{3}, x_{4}, \ldots\right), & t \in \infty .\end{cases}
$$

Again, by Lemma III-5, it is easy to check that $\psi$ is an invertible isotopy. Clearly $\psi_{t} \in L\left(F^{\infty}\right), t \in[0, \infty]$.

Lemma IV-2. Let $F$ be a TVS. Define $\lambda: C(F) \times l \rightarrow C(F)$ by $\lambda(f, t)(x)=$ $f(x)-t f(0)$. Then $\lambda$ is continuous.

Proof of IV-2. Let $\lambda\left(f, t_{0}\right) \in C(K, U)$, where $K$ is a compact subset of $F$ and $U$ is an open subset of $F$. Then, for each $k \in K, f(k)-t_{0} f(0) \in U$. Define $\sigma: F \times F \times I \rightarrow F$ by $\sigma(x, y, t)=x-t y$. Then $\sigma$ is continuous, and, for each $k \in K, \sigma\left(f(k), f(0), t_{0}\right) \in U$. Since $K$ is compact there are neighborhoods $\mathcal{O}, V, W$ of $f(K), f(0), t_{0}$, respectively, such that $\sigma(\Theta \times V \times W) \subset U$. Then

and

$$
\left(f, t_{0}\right) \in[C(K, \mathcal{O}) \cap C(\{0\}, V)] \times W,
$$

$$
\lambda([C(K, \mathcal{O}) \cap C(\{0\}, V)] \times W) \subset C(K, U) .
$$

Proof of Theorem II-4. By Corollary III-3 $F$ has the DLPP and there is a homeomorphism $\beta: F \rightarrow F^{\infty}$. We may assume $\beta(0)=(0,0, \ldots)$. By Lemma IV-1 and Theorem II-2 there is a contraction $\Psi: P\left(F^{\infty}\right) \times I \rightarrow P\left(F^{\infty}\right)$ of $P\left(F^{\infty}\right)$ to the identity map on $F^{\infty}$ such that $\Psi(g \circ f, t)=\Psi(g, t) \circ \Psi(f, t)$ and such that $\Psi$ simultaneously contracts $\mathcal{O P}\left(F^{\infty}\right), E P\left(F^{\infty}\right), C E P\left(F^{\infty}\right)$ and $H P\left(F^{\infty}\right)$. Let $\beta^{*}: C(F) \rightarrow C\left(F^{\infty}\right)$ be the homeomorphism $\beta^{*}(f)=\beta \circ f \circ \beta^{-1}$. Define $\lambda: C(F) \times I \rightarrow C(F)$ by $\lambda(f, t)=f-t f(0)$. By Lemma IV-2, $\lambda$ is continuous. Define $\Lambda: C(F) \times I \rightarrow C(F)$ by

$$
\Lambda(f, t)= \begin{cases}\lambda(f, 2 t), & t \in[0,1 / 2], \\ \left(\beta^{*}\right)^{-1} \circ \Psi\left(\beta^{*}(\lambda(f, 1)), 2 t-1\right), & t \in[1 / 2,1] .\end{cases}
$$

Then $\Lambda$ is the desired contraction. If $X$ is a TVS, then Theorem II-2 applies to $F=X^{\infty}$ immediately, and the homeomorphisms $\beta^{*}$ and $\left(\beta^{*}\right)^{-1}$ may be omitted. 
The last statement of Theorem II-4 then follows by checking, in the proof of Theoerm II-2, that $\Psi\left(L\left(X^{\infty}\right) \times I\right) \subset L\left(X^{\infty}\right)$.

Now let $\alpha: A \times F \rightarrow F$ be given, where $A \times F$ is a $k$-space, and let U: $A \times F \times I \rightarrow A \times F \times I$ be defined by $\mathscr{U}(a, x, t)=(a, \Lambda(\alpha(a,), t).(x), t)$. Then, on $A \times F \times[0,1 / 2]$,

$$
\mathcal{U}(a, x, t)=(a, \alpha(a, x)-2 t \alpha(a, 0), t),
$$

and this is clearly continuous. Define $\alpha^{\prime}: A \times F \rightarrow F$ by $\alpha^{\prime}(a, x)=\lambda(\alpha(a,), 1).(x)$ $=\alpha(a, x)-\alpha(a, 0)$. Define $\alpha^{\prime \prime}: A \times F^{\infty} \rightarrow F^{\infty}$ by $\alpha^{\prime \prime}(a, y)=\beta\left(\alpha^{\prime}\left(a, \beta^{-1}(y)\right)\right)$. Then both $\alpha^{\prime}$ and $\alpha^{\prime \prime}$ are continuous, and $\alpha^{\prime \prime}(a,(0,0, \ldots))=(0,0, \ldots) \in F^{\infty}$ for each $a \in A$. By Theorem II-3 the map $\gamma: A \times F^{\infty} \times[1 / 2,1] \rightarrow F^{\infty}$ given by $\gamma(a, x, t)=$ $\Psi\left(\alpha^{\prime \prime}(a, \cdot), 2 t-1\right)(x)$ is continuous. Thus, the map $A \times F \times[1 / 2,1] \rightarrow F$ given by $(a, x, t) \rightarrow \beta^{-1}(\gamma(a, \beta(x), t))$ is al so continuous. But $\beta^{-1}(\gamma(a, \beta(x), t))=$ $\Lambda(\alpha(a, \cdot), t)(x), t \in[1 / 2,1]$, and it follows that $\mathcal{O}$ is continuous.

Now suppose also that $\bar{\alpha}$ is an open embedding, resp. closed embedding, homeomorphism. Then $\bar{\alpha}^{\prime \prime}: A \times F^{\infty} \rightarrow A \times F^{\infty}$ defined by $\bar{\alpha}^{\prime \prime}(a, y)=\left(a, \alpha^{\prime \prime}(a, y)\right)$ is an open embedding, resp. closed embedding, homeomorphism. Hence, by Theorem II-3, $\bar{\gamma}: A \times F^{\infty} \times[1 / 2,1] \rightarrow A \times F^{\infty} \times[1 / 2,1]$ defined by $\bar{\gamma}(a, x, t)=$ $(a, \gamma(a, x, t), t)$ is an open embedding, resp. closed embedding, homeomorphism. It follows that $\mathcal{U}$ restricted to $A \times F \times[1 / 2,1]$ is an open embedding, resp. closed embedding, homeomorphism. It is routine to check this for $\mathfrak{Q}$ restricted to $A \times F \times[0,1 / 2]$.

V. Proof of Theorem II-6. Throughout this section $B$ denotes a separable, infinite-dimensional Banach space.

Lemma V-1. There is a continuous linear injection $\lambda: B^{*}\left(b^{*}\right) \rightarrow l_{2}$, where $l_{2}$ is a separable Hilbert space.

Remark. In [7], Richard Graff establishes the stronger result (his Lemma $2.33)$ that there is a continuous dense linear injection $\lambda: B^{*}\left(b^{*}\right) \rightarrow l_{2}$. The lemma was first suggested to the author, however, by David Elworthy, and the proof we give here is in large part due to him.

Proof of V-l. Let $l_{1}=\left\{\left\{x_{i}\right\}\left|x_{i} \in R, i=1,2, \ldots, \Sigma\right| x_{i} \mid<\infty\right\}$ with norm $\|x\|=\Sigma\left|x_{i}\right|$. Let $\beta: l_{2} \rightarrow l_{1}$ be defined by $\left\{x_{i}\right\} \rightarrow\left\{x_{i} \mid\left(2^{i}\right)\right\}$. Then $\beta$ is an injective, compact linear operator with dense range. By a theorem of Banach and Mazur [2], there is a continuous linear surjection $\alpha: l_{1} \rightarrow B$. Then $\alpha \circ \beta: l_{2} \rightarrow B$ is a compact, linear operator with dense range.

Identify $l_{2}^{*}$ with $l_{2}$ in the canonical way, and note that this identification identifies the weak-* topology of $l_{2}^{*}$ with the weak topology of $l_{2}$. Let 
$\lambda=(\alpha \circ \beta)^{*}: B^{*} \rightarrow l_{2}$. Then $\lambda$ is a compact [6, Theorem 2, p. 485], injective linear operator. Let $B^{*}\left(w^{*}\right)$ denote $B^{*}$ with its weak-* topology. For each positive integer $n$, let $B_{n}^{*}=\left\{x^{*} \in B^{*} \mid\left\|x^{*}\right\| \leq n\right\}$, and let $B_{n}^{*}\left(w^{*}\right)$ denote $B_{n}^{*}$ with the topology induced from $B^{*}\left(w^{*}\right)$. Since $B^{*}\left(b^{*}\right)=\operatorname{inj} \lim B_{n}^{*}\left(w^{*}\right)$, it is enough to show that $\lambda: B_{n}^{*}\left(w^{*}\right) \rightarrow l_{2}$ is continuous. By Lemma 3, p. 478 in [6] $\lambda: B^{*}\left(w^{*}\right)$ $\rightarrow l_{2}(w)$ is continuous, where $l_{2}(w)$ is $l_{2}$ with its weak topology. Let $A=$ $\lambda\left(B_{n}^{*}\left(w^{*}\right)\right) \subset l_{2}$, and let $A(w)$ denote $A$ with its induced weak topology. We then have that $\lambda: B_{n}^{*}\left(w^{*}\right) \rightarrow A(w)$ is continuous. Thus, we are done provided id: $A \rightarrow$ $A(w)$ is a homeomorphism (the first $A$ having the norm topology). This will be true if $A$ is compact. To see this, recall that $B_{n}^{*}\left(w^{*}\right)$ is compact by Alaoglu's theorem [6, p. 424]. Thus, $A(w)$ is also compact. But then, the norm closure of $A$, which is contained in the weak closure of $A$, must be $A$, so that $A$ is closed in $l_{2}$. Since $\lambda$ is a compact operator, $A$ is then also compact.

Following R. D. Anderson in [1] we make the following definition.

Definition. A closed subset $K$ of a space $X$ has Property $Z$ in $X$ (or is a $Z$-set in $X$ ) if for each homotopically trivial nonempty open set $U$ in $X, U \backslash K$ is nonempty and homotopically trivial.

Theorem (Anderson [1]). Every bomeomorphism between two Z.sets of $Q$ can be extended to a bomeomorphism of $Q$ itself.

We take the following definitions and theorem from C. Bessaga and A. Pelczyński in [3].

Definition. Given a convex subset $W$ of a Fréchet space, then

$$
\text { rint } W=\{w \in W \mid \text { if } x \in W \text {, then } w+\epsilon(w-x) \epsilon W \text { for some } \epsilon>0\} \text {. }
$$

It follows that if $0 \in$ rint $W$, then rint $W=\{c w \mid w \in W$ and $0 \leq c<1\}$.

Definition. $Q_{\text {odd }}=\left\{\left\{x_{i}\right\} \in Q \mid x_{i}=0\right.$ for even $\left.i\right\}$.

Theorem (Bessaga and Pelczyński [3]). Let $K$ be an infinite-dimensional, compact convex subset of a Fréchet space such that $0 \in$ rint $K$. Then for every $a \in(0,1)$ the set $a K$ is a Z-set in $K$, and the pair $(K, a K)$ is bomeomorpbic to to the pair $\left(Q, Q_{\text {odd }}\right)$.

Proof of Theorem II-6. For each positive integer $n$, let $B_{n}^{*}\left(b^{*}\right)$ denote $B_{n}^{*}=$ $\left\{x^{*} \in B^{*} \mid\left\|x^{*}\right\| \leq n\right\}$ with its induced $b^{*}$ topology. Note that restricted to $B_{n}^{*}$ the $b^{*}$ topology agrees with the weak-* topology. By Lemma V-1 there is a continuous linear injection $\lambda: B^{*}\left(b^{*}\right) \rightarrow l_{2}$. Fix $n$. By Alaoglu's theorem [6, p. 424], $B_{n}^{*}\left(b^{*}\right)$ is compact, so $\lambda$ restricted to $B_{n}^{*}\left(b^{*}\right)$ is a homeomorphism. Thus, the pair $\left(B_{n+1}^{*}\left(b^{*}\right), B_{n}^{*}\left(b^{*}\right)\right)$ is homeomorphic to the pair $\left(\lambda\left(B_{n+1}^{*}\left(b^{*}\right)\right), \lambda\left(B_{n}^{*}\left(b^{*}\right)\right)\right)$. The latter pair is in turn, by the theorem of Bessaga and Pelczyński above, homeomorphic 
to $\left(Q, Q_{\text {odd }}\right)$. Since $Q_{\text {odd }}$ has Property $Z$ in $Q$ (this follows, for example, from Theorem 9.1 of [1]) it follows that each $B_{n}^{*}\left(b^{*}\right)$ is homeomorphic to $Q$ and has Property $Z$ in $B_{n+1}^{*}\left(b^{*}\right)$.

We now inductively define homeomorphisms $b_{n}: B_{n}^{*}\left(b^{*}\right) \rightarrow Q^{n}$ such that $b_{n+1}$ extends $b_{n}, b_{n}$ regarded as a homeomorphism onto $Q^{n} \times\{0\} \subset Q^{n+1}$. In this way we obtain a homeomorphism of direct limits:

$$
b: B^{*}\left(b^{*}\right)=\operatorname{inj} \lim B_{n}^{*}\left(b^{*}\right) \rightarrow Q^{\infty}=\operatorname{inj} \lim Q^{n} .
$$

Let $b_{1}$ be any homeomorphism of $B_{1}^{*}\left(b^{*}\right)$ onto $Q^{1}$. Let a homeomorphism $b_{n}: B_{n}^{*}\left(b^{*}\right) \rightarrow Q^{n}$ be given. Since $B_{n}^{*}\left(b^{*}\right)$ is a $Z$-set in $B_{n+1}^{*}\left(b^{*}\right)$ and $Q^{n}$ is a $Z$-set in $Q^{n+1}$ (e.g. by Theorem 9.1 of [1]) the theorem of Anderson given above says there is a homeomorphism $b_{n+1}: B_{n+1}^{*}\left(b^{*}\right) \rightarrow Q^{n+1}$ extending $b_{n}$, as required.

Finally, the second statement of Theorem II- 6 follows from the observation that the natural isomorphism $l_{2} \rightarrow l_{2}^{*}$ identifies the bounded weak topology on $l_{2}$ with the $b^{*}$ topology on $l_{2}^{*}$ 。

\section{REFERENCES}

1. R. D. Anderson, On topological infinite deficiency, Michigan Math. J. 14 (1967), 365-383. MR $35 \# 4893$.

2. S. Banach and S. Mazur, Zur Theorie der linearen Dimension, Studia Math. 4 (1933), 100-112.

3. C. Bessaga and A. Pelczyński, The estimated homogeneous collections and skeletons, and their applications to the topological classification of linear metric spaces and convex sets, Fund. Math. 69 (1970), 153-190. MR 42 \#8227.

4. T. A. Chapman, Locally-trivial bundles and microbundles with infinite-dimensional fibers, Proc. Amer. Math. Soc. 37 (1973), 595-602.

5. J. Dugundji, Topology, Allyn and Bacon, Boston, Mass., 1966. MR 33 \#1824.

6. N. Dunford and J. T. Schwartz, Linear operators. I: General theory, Pure and Appl. Math., vol. 7, Interscience, New York, 1958. MR 22 \#8302.

7. R. A. Graff, Elements of local non-linear functional analysis, Thesis, Princeton University, Princeton, N. J., 1972.

8. R. S. Palais, Lectures on the differential topology of infinite-dimensional manifolds, Brandeis University, Waltham, Mass., 1964-1965.

9. P. L. Renz, The contractibility of the homeomorphism group of some product spaces by Wong's method, Math. Scand. 28 (1971), 182-188.

DEP ARTMENT OF MATHEMATICS, CORNELL UNIVERSITY, ITHACA, NEW YORK 14850

Current address: Department of Mathematics, Vanderbilt University, Nash ville, Tennessee 37235 